\title{
Numerical study of drop behavior in a pore space
}

\author{
Fenglei Huang ${ }^{1,2}$, Zhe Chen ${ }^{2}$, Zhipeng $\mathrm{Li}^{1}$, Zhengming Gao ${ }^{1 *}$, J.J. Derksen ${ }^{3}$, Alexandra Komrakova ${ }^{2 *}$ \\ ${ }^{1}$ School of Chemical Engineering, Beijing University of Chemical Technology, Beijing, China \\ ${ }^{2}$ Department of Mechanical Engineering, University of Alberta, Edmonton, Canada \\ ${ }^{3}$ School of Engineering, University of Aberdeen, Aberdeen, UK \\ * Corresponding author. E-mail address: gaozm@mail.buct.edu.cn (Zhengming Gao), komrakov@ualberta.ca (Alexandra Komrakova).
}

\begin{abstract}
Deformation and breakup of a liquid drop immersed in another immiscible liquid and flowing through a single pore has been studied numerically using a conservative phase-field lattice Boltzmann method. Several benchmarks were conducted to validate the code, including the recovery of Laplace pressure, the layered flow of two immiscible liquids, and the implementation of wetting boundary conditions on a curved surface. Gravity-driven motion of a drop through the pore space was qualitatively compared to the available experimental results. Quantitative assessment of the pressure field across the interface of the moving and deforming drop was performed. Our results show that high Weber number due to low surface tension and low Reynolds number due to low velocity of the continuous liquid promote drop breakage. More viscous drops break easier than less viscous drops. We present the phase charts (Weber vs capillary number) and the critical conditions (Weber as a function of Reynolds number) of drop breakage.
\end{abstract}

Keywords: Pore space; Liquid-liquid dispersion; Drop breakup; Phase-field method

\section{Highlights:}

- The phase-field method to simulate liquid-liquid systems is verified and validated.

- The behavior of a single drop moving through a pore space is studied numerically.

-Low velocity of the continuous liquid promote drop breakage.

- More viscous drops break easier than less viscous drops.

- Drop behavior is presented on We vs $C a$ and $W e$ vs $R e$ graphs. 


\section{Graphical Abstract}
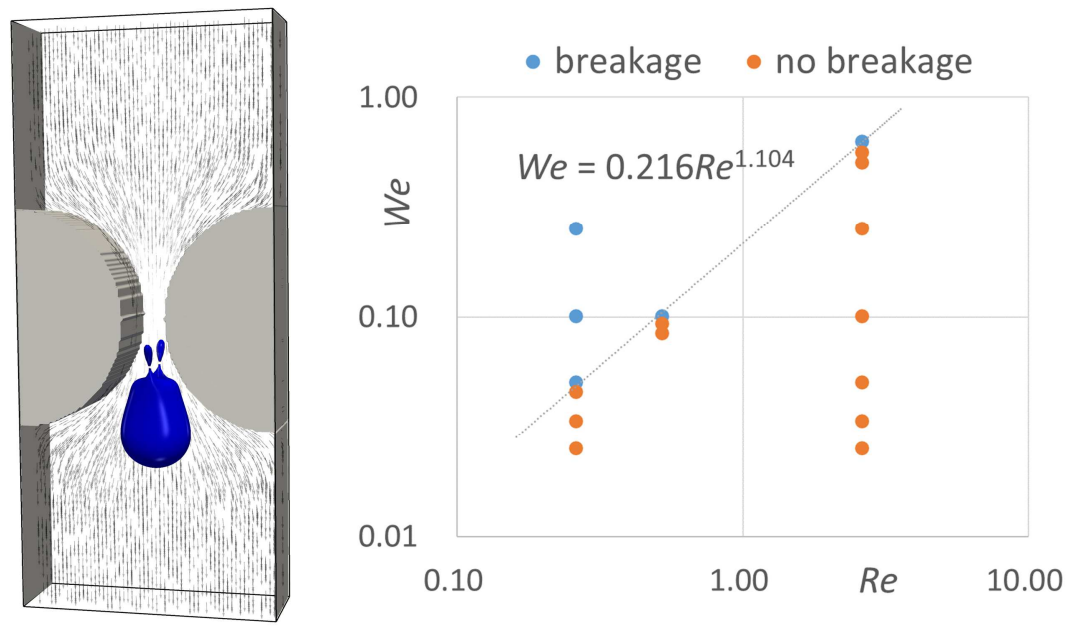

\section{Introduction}

Multiphase flow in porous media is ubiquitous in nature and engineering (Adler and Brenner, 1988; Jacob, 2018):

some examples include groundwater flow and flow in geothermal engineering, drainage, and irrigation in agricultural engineering, the flow of oil, water and gas in oil reservoirs, flow in reactors in chemical engineering, flow in fuel cells, filters and membranes (Telmadarreie et al., 2016; Li et al., 2018; Perazzo et al., 2018; Kim et al., 2020; Lee et al., 2020). In such systems, two key phenomena occur at the microscopic or pore-scale that affect the macroscopic behavior of the system: 1) motion of deformable interface between the fluids (e.g. gas-liquid dispersion or liquid-liquid emulsion); and 2) interaction of this interface with the surrounding solid phase (contact line motion). A thorough understanding of pore-scale phenomena is crucial to make well-guided decisions on entire system control and efficient operation. For that reason, numerous experimental and numerical studies focus on the investigation of multiphase flow in a simplified porous medium and single pore.

Single drops motion in idealized pore geometries were studied experimentally and numerically. The pore geometries range from wavy-wall capillary tubes to single pores formed by spherical solid particles. The common goal for the majority of these studies is to identify the effect of the geometry on drop motion and breakup. Olbricht and Leal (1983) 
experimentally studied the creeping flow of an immiscible Newtonian drop through a horizontal circular tube with a periodically varying diameter in the axial direction (wavy-wall tube). The contribution of the drop to the local pressure gradient and the velocity of the drop relative to the average two-phase velocity were measured and then correlated to the time-dependent change of the drop shape. Drop breakup was also observed and analyzed primarily qualitatively.

The buoyancy-driven motion of viscous drops and gas bubbles in a vertical periodically constricted capillary was studied experimentally by Hemmat and Borhan (1996) to examine the role of capillary geometry on drop deformation and breakup. The authors reported the measurements of the average rise velocity of drops and their shapes for a wide range of governing parameters and outlined mechanisms of drop breakup.

Olgac et al. (2006) performed a numerical study of buoyancy-driven viscous drops though sinusoidally constricted capillaries using a finite-volume/front-tracking method. After validation of the method by comparison to the experimental results of Hemmat and Borhan (1996), the authors studied the effects of the drop size, the channel geometry, and Bond number on the motion and breakup of viscous drops in constricted capillaries. Different breakup mechanisms were examined and analyzed quantitatively.

The behavior of a non-wetting drop flowing through a pore formed by two spherical solid particles was numerically studied by Hellou and Vo (2015) using a volume of fluid method. The authors described the deformation process of the viscous drop in viscous fluid that was quiescent or moving with uniform velocity in the direction of gravity. A comparison to experimental analysis was performed to validate the method. Drop breakup conditions were defined as a function of the size of the constriction.

A review on motion of deformable non-wetting liquid drops flowing through well-defined porous media at zero Reynolds number was published by Zinchenko and Davis (2017). The authors considered theoretical solutions and rigorous hydrodynamical simulations for both pore and large scale. 
Ansari et al. $(2018,2019)$ conducted an experimental study of liquid-liquid flow through a pore to determine the pressure field by analysis of the velocity field and shape of the drop obtained by the micro particle shadow velocimetry $(\mu-P S V)$.

Patel et al. (2019) numerically studied the dynamics of a bubble rising in a vertical sinusoidal wavy channel using a dual grid level set method coupled with a finite volume-based discretization of the Navier-Stokes equations. Bubble deformation and breakup was correlated to a Reynolds number, Bond number, and the amplitude of the channel wall.

The flow of dispersed drops and bubbles also finds numerous applications in microfluidic devices with constrictions and contractions. For instance, as discussed by Zheng et al. (2020), microfluidic channels with constrictions provide an effective and reliable way to manipulate fluid particles especially in areas such as biology and clinical diagnostics.

A review of bubbles and drops flowing in different microfluidic geometrical elements was given by Cerdeira et al. (2020). Jensen et al. (2004) and Chio et al. (2006) studied the dynamics of gas bubbles moving in liquid-filled microchannels with contractions. Flow patterns and frictional pressure drop in a microchannel with alternating expansions and constrictions were experimentally investigated by Chai et al. (2015) for gas-liquid systems.

To the best of our knowledge, there is a limited number of published experimental and numerical studies that report on the behavior of liquid drops moving in another immiscible liquid through contracted microchannels or microchannels with constrictions.

A numerical parametric study of drop deformation through a microfluidic contraction was studied by Harvie et al. (2005) using a transient volume of fluid finite volume algorithm. The authors covered the parameter ranges representative of micro-sized liquid-liquid systems. The effects of the Reynolds number, interfacial tension, and the dispersed to continuous phase viscosity ratio on the deformation of the drop passing an axisymmetric contraction were reported. The phase charts (capillary number as a function of Weber number at different Ohnesorge numbers) of drop 
deformation and breakup were presented. Later the study was extended to cover low viscosity Newtonian drops (Harvie et al., 2006).

Mulligan et al. (2011) studied the effect of confinement-induced shear on drop deformation and breakup in microfluidic extensional flows. The behavior of de-ionized water drops in oil was considered experimentally. Conditions to produce drops after breakage much smaller than the parent drop were outlined.

Chung et al.(2008) performed a numerical study on the effect of viscoelasticity on the drop in a planar contraction/expansion microchannel using a finite element front tracking method with the goal to propose the strategy to control the drop shape.

Izbassarov and Muradoglu (2016) computationally studied two-phase viscoelastic systems in a pressure-drive flow in an axisymmetric channel with a sudden contraction and expansion using a finite-difference/front-tracking method. Drop dynamics were examined in a wide range of governing parameters.

Zheng et al. (2020) carried out numerical simulations of bubbles and drops flowing in a rectangular channel with obstruction focusing on the disruption of the wake downstream of the obstruction by the fluid particle and the trajectory of that particle.

As can be seen from the literature review above, most of the available numerical studies are performed in the axisymmetric formulation. Three-dimensional transient simulations of liquid-liquid systems in pore geometry with curved boundaries are still missing and should be performed as a necessary validation step towards modeling of multiphase flow in realistic porous media.

In this work, we numerically study the time-dependent motion of Newtonian liquid drops flowing through a single pore in a three-dimensional microchannel in the continuous flow of another immiscible Newtonian liquid. Our main goal is to outline the conditions of drop breakup when it passes through a pore throat. When the drop breaks, satellite and sub-satellite drops with a size significantly smaller than the mother drop size can be generated. For some processes, for 
instance, when it is eventually necessary to separate the immiscible liquids or when the drop size distribution should be monodisperse, the production of small fragments is highly undesirable. A map that indicates the conditions when drop breakage occurs is necessary to better control the system of two immiscible liquids flowing through porous media.

We use a phase-field lattice Boltzmann method to perform the simulations. To verify and validate our numerical code, we performed a series of benchmark cases: recovery of the Laplace pressure of a spherical drop, the layered flow of two immiscible liquids with different viscosities, recovery of the contact angle at a curved surface, the gravity-driven motion of a drop in ambient liquid in a channel, and the gravity-driven drop motion in ambient liquid through a pore space. Then the code was applied to investigate the main subject of this study.

The rest of the paper is organized as follows: Section 2 contains the governing equations, the numerical method to solve the equations, and the wetting boundary conditions scheme. The validation of the numerical method is shown in Section 3. Results and discussion are given in Section 4. Finally, the conclusions are presented in Section 5.

\section{Mathematical Model}

\section{Governing Equations}

A conservative phase-field model proposed by Mitchell et al. (2018) is used in this study to simulate the flow of two immiscible liquids. The phase-field model corresponds to a class of diffuse interface approaches where the sharp interface between the liquids is replaced by a smooth region of finite width where fluid properties vary continuously, but rapidly. An order parameter, i.e., the phase-field $\phi$, describes the phase transition: its values are constant in the bulk phases and vary smoothly across the interface region. A phase-field equation that describes the evolution of $\phi$ in the entire computational domain is derived based on thermodynamically consistent theories. The phase-field model does not require any explicit procedures to track the changes in the topology of the interface. This reduces programming complexity and decreases computational time related to the explicit reconstruction of the interface as in sharp interface methods. Any topological changes of the interface are handled naturally due to thermodynamic mechanisms involved. In 
this study, we use the conservative phase-field model proposed by Chiu and Lin (2011) based on the work of Sun and Beckermann (2007).

A system of coupled equations which are the continuity, Navier-Stokes, and phase-field equation governs the dynamics of a two-phase system of incompressible immiscible fluids:

$$
\begin{gathered}
\nabla \cdot \boldsymbol{u}=0 \\
\rho\left(\frac{\partial \boldsymbol{u}}{\partial t}+\boldsymbol{u} \cdot \nabla \boldsymbol{u}\right)=-\nabla p+\nabla \cdot\left(\mu\left[\nabla \boldsymbol{u}+(\nabla \boldsymbol{u})^{\mathrm{T}}\right]\right)+\boldsymbol{F} \\
\frac{\partial \phi}{\partial t}+\nabla \cdot(\phi \boldsymbol{u})=\nabla \cdot M\left(\nabla \phi-\frac{1-4\left(\phi-\phi_{0}\right)^{2}}{\xi} \boldsymbol{n}\right)
\end{gathered}
$$

where $\boldsymbol{u}$ is the macroscopic velocity, $\rho$ is the density, $t$ is the time, $p$ is the pressure; $M$ is the mobility; $\xi$ is the interface thickness, and $\boldsymbol{n}=\frac{\nabla \phi}{|\nabla \phi|}=$ is the unit vector normal to the interface. The phase-field $\phi$ takes two extreme values in the bulk phases: $\phi_{\mathrm{H}}$ and $\phi_{\mathrm{L}}$, representing the heavy and light phases, respectively. The value of $\phi$ at the interface is given by:

$$
\phi_{0}=\left(\phi_{\mathrm{H}}+\phi_{\mathrm{L}}\right) / 2
$$

In this work, we set $\phi_{\mathrm{H}}=1$ for the heavy phase and $\phi_{\mathrm{L}}=0$ for the light phase, which gives $\phi_{0}=0.5$.

A simple linear interpolation is employed to calculate the local density $\rho$ from the phase field,

$$
\rho=\rho_{\mathrm{L}}+\phi\left(\rho_{\mathrm{H}}-\rho_{\mathrm{L}}\right)
$$

where $\rho_{\mathrm{L}}$ and $\rho_{\mathrm{H}}$ are the densities of the light and heavy phase, respectively.

The volumetric forcing term in Eq. (2) is defined as $\boldsymbol{F}=\boldsymbol{F}_{\mathbf{b}}+\boldsymbol{F}_{\mathbf{s}}$, where $\boldsymbol{F}_{\mathbf{b}}=\rho \boldsymbol{g}$ ( $\boldsymbol{g}$ is the gravitational acceleration) and $\boldsymbol{F}_{\mathrm{s}}$ are the body and surface tension forces, respectively. The surface tension $\boldsymbol{F}_{\mathrm{s}}$ is defined as

$$
\boldsymbol{F}_{\mathbf{s}}=\mu_{\phi} \nabla \phi
$$

with the chemical potential $\mu_{\phi}$ being defined as (Zu and He, 2013):

$$
\mu_{\phi}=1.5 \sigma\left[\frac{32 \phi(\phi-1)(\phi-0.5)}{\xi}-\xi \nabla^{2} \phi\right]
$$

where $\sigma$ is the surface tension. 


\section{Lattice Boltzmann Formulation}

The governing macroscopic equations (1)-(3) were solved using the lattice Boltzmann method in the formulation proposed by Mitchell et al. (2018). Two particle populations are necessary to solve the system: $f_{\alpha}(\boldsymbol{x}, t)$ is used to solve the continuity and the momentum equations, and the second function $g_{\alpha}(x, t)$ is used to solve the phase-field equation. These particle populations represent the density of fictitious particles with discrete velocity $\boldsymbol{e}_{\alpha}$ at the position $\boldsymbol{x}$ and time $t$. The discrete velocity $\boldsymbol{e}_{\alpha}$ together with the corresponding weighting coefficients $\omega_{\alpha}$ form the velocity set $\left(\boldsymbol{e}_{\alpha}, \omega_{\alpha}\right)$. For the $f$ population, we use a $\mathrm{D} 3 \mathrm{Q} 27$ set which indicates that the number of spatial dimensions is $\mathrm{D}=3$ and the velocity set involves $\mathrm{Q}=27$ velocity directions $(\alpha=0: 26)$. The D3Q15 set is used for the $g$ population. The lattice velocities $\boldsymbol{e}_{\alpha}$ and weights $\omega_{\alpha}$ used in this work are given in Appendix A.

The lattice Boltzmann equations discretized in the velocity space, physical space and time are given as follows:

$$
\begin{gathered}
f_{\alpha}\left(\boldsymbol{x}+\boldsymbol{e}_{\alpha} \delta t, t+\delta t\right)=f_{\alpha}(\boldsymbol{x}, t)-\left(\mathbf{M}^{-1} \hat{\mathbf{S}} \mathbf{M}\right)_{\alpha \beta}\left[f_{\beta}(\boldsymbol{x}, t)-\bar{f}_{\beta}^{e q}(\boldsymbol{x}, t)\right]+F_{\alpha}(\boldsymbol{x}, t) \\
g_{\alpha}\left(\boldsymbol{x}+e_{\alpha} \delta t, t+\delta t\right)=g_{\alpha}(\boldsymbol{x}, t)-\frac{g_{\alpha}(\boldsymbol{x}, t)-\bar{g}_{\alpha}^{e q}(\boldsymbol{x}, t)}{\tau_{\phi^{+1 / 2}}}+F_{\alpha}^{\phi}(\boldsymbol{x}, t)
\end{gathered}
$$

These equations state that the particle populations move with the velocity $\boldsymbol{e}_{\alpha}$ to the neighboring sites $\boldsymbol{x}+\boldsymbol{e}_{\alpha} \delta t$ at the next time step $t+\delta t$ (streaming). The collision of particles on a given site is governed by the collision operator (second term on the right-hand side of equations (8) and (9)) which redistributes particles among the populations. A weighted multiple-relaxation-time (WMRT) collision operator is used for the $f$ population since it improves the isotropy, decreases the spurious velocity, and increases the model accuracy (Fakhari et al., 2017a). A single-relaxation-time Bhatnagar-Gross-Krook (BGK) (Bhatnagar et al., 1954) collision operator is used for the $g$ population. The physical mesh step $\delta x$ and the time step $\delta t$ denote a space and time resolution in lattice units which is an artificial set of units scaled such that $\delta x=1$ and $\delta t=1$, and $c=\delta x / \delta t$ is the lattice speed. To convert lattice units to a physical space in SI units we will match the governing dimensionless numbers. 
The rest of the quantities in equations (8) and (9) are defined as follows. The shifted equilibrium population due to forcing is

$$
\bar{f}_{\alpha}^{e q}=f_{\alpha}^{e q}-\frac{1}{2} F_{\alpha}
$$

with the equilibrium population

$$
f_{\alpha}^{e q}=\omega_{\alpha}\left[p^{*}+\left(\frac{\boldsymbol{e}_{\alpha} \cdot \boldsymbol{u}}{c_{s}^{2}}+\frac{\left(\boldsymbol{e}_{\boldsymbol{\alpha}} \cdot \boldsymbol{u}\right)^{2}}{2 c_{s}^{4}}-\frac{\boldsymbol{u} \cdot \boldsymbol{u}}{2 c_{s}^{2}}\right)\right]
$$

where $p^{*}=p / \rho c_{s}^{2}$ is the normalized pressure with $c_{s}$ is the speed of sound in this system, $c_{s}=c / \sqrt{3}$.

The equilibrium distribution $\bar{g}_{\alpha}^{e q}$ is also shifted including the forcing term as

$$
\bar{g}_{\alpha}^{e q}(\boldsymbol{x}, t)=g_{\alpha}^{e q}-\frac{1}{2} F_{\alpha}^{\phi}
$$

where

$$
g_{\alpha}^{e q}=\phi \omega_{\alpha}\left(1+\frac{\boldsymbol{e}_{\alpha} \cdot \boldsymbol{u}}{c_{s}^{2}}+\frac{\left(\boldsymbol{e}_{\boldsymbol{\alpha}} \cdot \boldsymbol{u}\right)^{2}}{2 c_{S}^{4}}-\frac{\boldsymbol{u} \cdot \boldsymbol{u}}{2 c_{S}^{2}}\right)
$$

In Eq. (8), $\mathbf{M}$ is the WMRT transformation matrix given in Appendix B; and $\hat{\mathbf{S}}$ is the diagonal relaxation matrix which is given as

$$
\widehat{\mathbf{S}}=\operatorname{diag}\left(1,1,1,1, s_{v}, s_{v}, s_{v}, s_{v}, s_{v}, 1, \ldots, 1\right)
$$

where $s_{v}=(\tau+0.5)^{-1}$ is the relaxation parameter related to the hydrodynamic relaxation time $\tau$. The relaxation time could be calculated by many forms of interpolations (Fakhari et al., 2017b). In this study, $\tau$ is calculated by a simple linear interpolation

$$
\tau=\tau_{L}+\phi\left(\tau_{H}-\tau_{L}\right)
$$

where $\tau_{H}$ and $\tau_{L}$ are the bulk relaxation times in the heavy and light fluids, respectively. The dynamic viscosity $\mu$ is represented as 


$$
\mu=\rho v=\rho \tau c_{s}^{2}
$$

where $v$ is the kinematic viscosity.

For the $g$ population, $\tau_{\phi}=M / c_{s}^{2}$ is the relaxation time of the phase-field model.

The hydrodynamic forcing term $F_{\alpha}(\boldsymbol{x}, t)$ in Eq. (8) is calculated as

$$
F_{\alpha}(\boldsymbol{x}, t)=\omega_{\alpha} \frac{\boldsymbol{e}_{\alpha} \cdot \boldsymbol{F}}{\rho c_{s}^{2}}
$$

where the total force term is

$$
\boldsymbol{F}=\boldsymbol{F}_{\mathrm{b}}+\boldsymbol{F}_{\mathrm{s}}+\boldsymbol{F}_{\mathrm{p}}+\boldsymbol{F}_{\mu}
$$

The pressure force $\boldsymbol{F}_{\mathrm{p}}$ is determined by (Fakhari et al., 2017b)

$$
\boldsymbol{F}_{\mathrm{p}}=-p^{*} c_{s}^{2}\left(\rho_{H}-\rho_{L}\right) \nabla \phi
$$

and the viscous force $\boldsymbol{F}_{\mu}$ is (Fakhari et al., 2017b)

$$
\boldsymbol{F}_{\mu}=v\left(\rho_{H}-\rho_{L}\right)\left[\nabla \boldsymbol{u}+(\nabla \boldsymbol{u})^{\mathrm{T}}\right] \cdot \nabla \phi
$$

where the derivative of velocity is recovered from the second moment of the hydrodynamic distribution function as

$$
\boldsymbol{F}_{\mu, i}=-\frac{v\left(\rho_{H}-\rho_{L}\right)}{c_{S}^{2}}\left[e_{\alpha, i} e_{\alpha, j}\left(\mathbf{M}^{-1} \hat{\mathbf{S}} \mathbf{M}\right)_{\partial, \beta}\left(f_{\beta}-f_{\beta}^{e q}\right)\right] \partial_{j} \phi
$$

where index $\alpha$ and $\beta$ represent the $\alpha$ th and $\beta$ th direction for the lattice structure and index $i$ and $j$ represent the index Cartesian coordinates directions $x, y, z$. Einstein summation convention is applied in Eq. (21).

The forcing term in Eq. (9) is given as

$$
F_{\alpha}^{\phi}(\boldsymbol{x}, t)=\delta t \frac{4 \phi(1-\phi)}{\xi} \omega_{\alpha} \boldsymbol{e}_{\alpha} \cdot \mathbf{n}
$$

The particle distribution functions are defined such that the following summations over all directions $\alpha$, at a single lattice point, give the local normalized pressure, local fluid velocity and local phase-field parameter, respectively: 


$$
\begin{gathered}
p^{*}=\sum_{\alpha} f_{\alpha} \\
\boldsymbol{u}=\sum_{\alpha} f_{\alpha} \boldsymbol{e}_{\boldsymbol{\alpha}}+\frac{\boldsymbol{F}}{2 \rho} \\
\phi=\sum_{\alpha} g_{\alpha}
\end{gathered}
$$

where $\boldsymbol{u}$ needs to be updated after the pressure.

The gradients and Laplacian of the phase-field variable $\phi$ are determined using all the neighboring nodes by the results of the conservative LBE for solving the continuity equation and the phase-field which will be introduced in the next section and are given as (Mitchell et al., 2018)

$$
\begin{gathered}
\nabla \phi=\frac{c}{c_{S}^{2} \delta x} \sum_{\alpha=0}^{26} \boldsymbol{e}_{\alpha} \omega_{\alpha} \phi\left(\boldsymbol{x}+\boldsymbol{e}_{\boldsymbol{\alpha}} \delta t, t\right) \\
\nabla^{2} \phi=\frac{2 c^{2}}{c_{S}^{2}(\delta x)^{2}} \sum_{\alpha=0}^{26} \omega_{\alpha}\left[\phi\left(\boldsymbol{x}+\boldsymbol{e}_{\boldsymbol{\alpha}} \delta t, t\right)-\phi(\boldsymbol{x}, t)\right]
\end{gathered}
$$

\section{Wetting Boundary Conditions}

In this study, a single pore throat is represented by two curved (cylindrical) surfaces. To investigate the drop behavior as it passes through the pore and interacts with the wall, we need to define the three-phase contact angle on flat and curved boundaries. To obtain a specified contact angle at a solid wall, the boundary condition proposed by Jacqmin (2000) is used here:

$$
\left.\widehat{\boldsymbol{n}}_{w} \cdot \nabla \phi\right|_{x_{w}}=\Theta \phi_{w}\left(1-\phi_{w}\right)
$$

where $\hat{\mathrm{n}}_{\mathrm{w}}$ is the unit vector outward normal to the wall, $\phi_{w}$ is the value of the phase-field parameter at the solid wall and $\Theta$ is a term related to the equilibrium contact angle $\theta$ which is given by:

$$
\Theta=-\sqrt{\frac{2 \varepsilon}{\kappa}} \cos \theta
$$

where coefficients $\varepsilon$ and $\kappa$ are related to the interfacial thickness $\xi$ and the surface tension $\sigma$ by $\varepsilon=12 \sigma / \xi$ and $\kappa=3 \sigma \xi / 2 \quad$ (Fakhari et al., 2017b). 


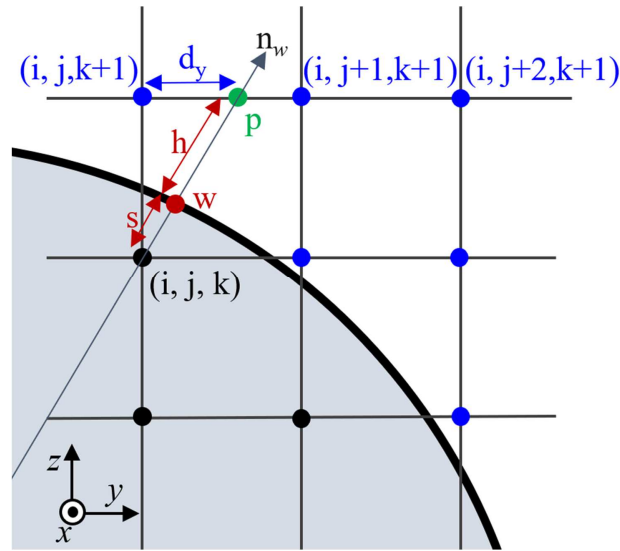

(a)

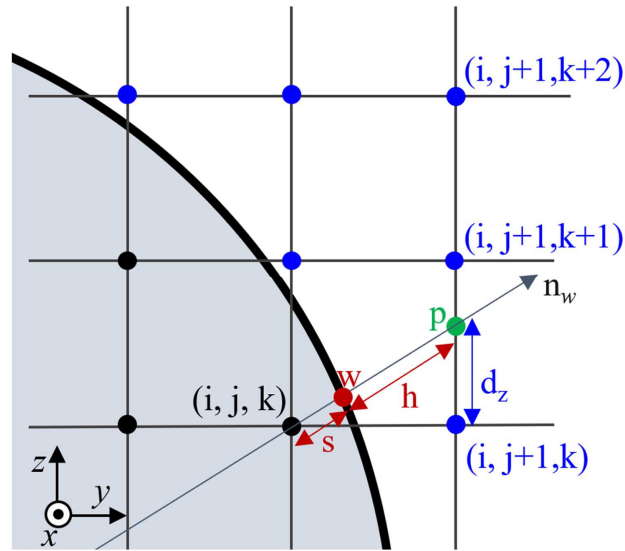

(b)

Fig. 1. Wetting boundary conditions at a curved boundary when the slope's magnitude of the vector normal to the boundary is (a) greater than one (b) less than one (adapted from (Fakhari and Bolster, 2017)).

To impose the wetting boundary conditions on a curved wall, we need to apply Eq. (28) and calculate the value of $\phi_{w}$ at the wall. We used unidirectional interpolations to calculate the unknown value of the phase-field parameter at the boundary node $\left(\phi_{i, j, k}\right)$, represented by a black point in Fig. 1. If the slope's magnitude of the vector normal to the boundary is greater than one, as shown in Fig. 1(a), then a linear interpolation is conducted in the $y$-direction; otherwise, the interpolation is conducted in the $z$-direction as shown in Fig. 1(b), to obtain the $\phi_{p}$ value at point $p$ with green color. Finally, the value of $\phi_{i, j, k}$ is calculated as (Fakhari and Bolster, 2017):

$$
\phi_{i, j, k}=\frac{s+h}{2 a h}\left(1+a-\sqrt{(1+a)^{2}-4 a \phi_{p}}\right)-\frac{s}{h} \phi_{p}, a=h \Theta \neq 0\left(\theta \neq 90^{\circ}\right)
$$

where $\mathrm{s}=\left|x_{w}-x_{i, j, k}\right|$ is the distance between the solid wall and the boundary node.

\section{Model Validation}

\section{Laplace pressure}

In this benchmark case, we assess the ability of the method to accurately recover the pressure change across the interface of the spherical drop. The pressure change can be described by the Laplace law:

$$
\Delta p=\frac{2 \sigma}{r}
$$


where $\Delta p$ is the pressure difference and $r$ is the radius of the drop. A series of simulations were performed to validate the pressure difference over the interface for different drop radii and values of surface tension. The simulations were performed in a fully-periodic cubic domain with an edge length of 64 lattice units (lu). The interface thickness $\xi$ was set to 6 lu. Drop and the surrounding liquid had matching density and viscosity. The drop was placed at the center of the domain and let to equilibrate. The maximum spurious velocities in these simulations are at the order of $10^{-6}$, so we can ignore their effects. The pressure drop estimated using Eq. (31) and predicted numerically for drop radii $r$ (10, 16, and 20 lu) and surface tension $\sigma(0.01,0.02$, and $0.04 \mathrm{lu})$ are shown in Fig. 2. The maximum deviation between the numerical and analytical results is less than $3 \%$.

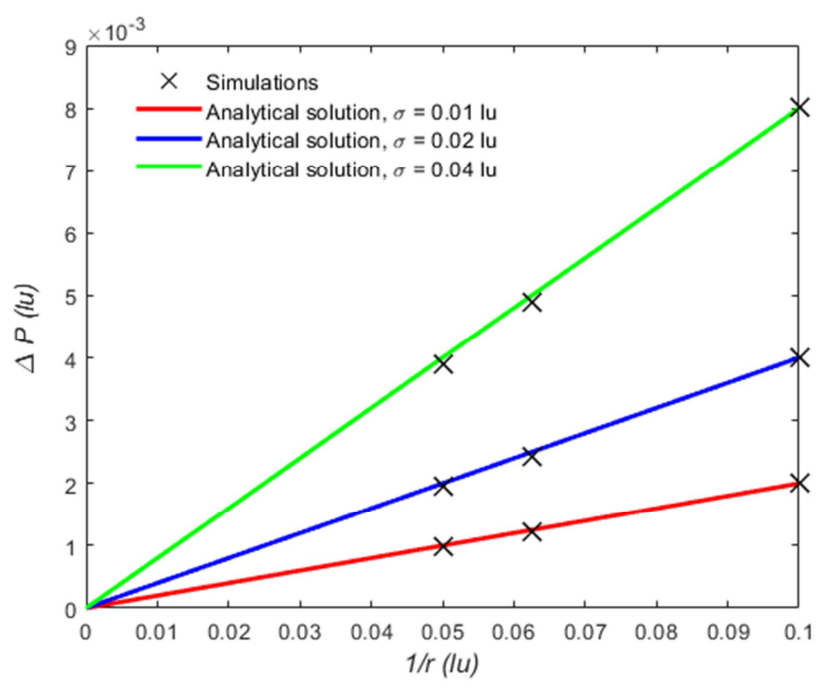

Fig. 2. The pressure change across the interface of the drop $\Delta p$ for different drop radii and values of surface tension

\section{Layered Flow of Immiscible Fluids}

The layered flow of two immiscible liquids was selected to test the capability of the method to handle high viscosity ratios between the liquids (Komrakova et al., 2015). The flow was simulated in a pseudo-2D simulation domain of $3 \times 3 \times 40$ lu, where 40 lu is the distance between two solid walls. Liquid 1 occupies the lower half of the channel, Liquid 2 the upper half. The no-slip bounce-back boundary conditions were applied at the top and bottom walls, and periodical boundary conditions for the rest of the boundaries. We applied a body force of $10^{-7}$ lu in the entire domain to create a pressure gradient in the flow direction. The viscosity ratios were $\eta=\left(\mu_{1} / \mu_{2}\right)=1,10,100$ and 1000 . 
The analytical solution for the velocity in the channel as a function of the wall normal coordinate $Z$ was calculated from the Navier-Stokes equation (Schulz et al., 2019):

$$
\begin{array}{lc}
u=\frac{d p}{d y}\left[\frac{1}{2 \mu_{1}} z^{2}+\frac{h\left(\mu_{2}-\mu_{1}\right)}{2 \mu_{1}\left(\mu_{1}+\mu_{2}\right)} z-\frac{h^{2}}{\mu_{1}+\mu_{2}}\right] & -h \leq z \leq 0 \\
u=\frac{d p}{d y}\left[\frac{1}{2 \mu_{2}} z^{2}+\frac{h\left(\mu_{2}-\mu_{1}\right)}{2 \mu_{2}\left(\mu_{1}+\mu_{2}\right)} z-\frac{h^{2}}{\mu_{1}+\mu_{2}}\right] & 0 \leq z \leq h
\end{array}
$$

where $\frac{d p}{d y}$ is the pressure gradient, $2 h$ is the channel height, $y$ is the direction of the flow and $\mu_{1}$ and $\mu_{2}$ are the dynamic viscosities of the liquids that have the same density $\left(\rho_{1}=\rho_{2}=1.0 \mathrm{lu}\right)$.

The comparison between the analytical and simulated results is shown in Fig. 3. The velocity profiles obtained numerically coincide with the analytical solution. The maximum deviation between the numerical and analytical results is less than $1 \%$. This demonstrates that the method can be used to simulate the flow of liquids with the viscosity ratio up to 1000. The L2 convergence analysis at $\eta=100$ is shown in Appendix C.
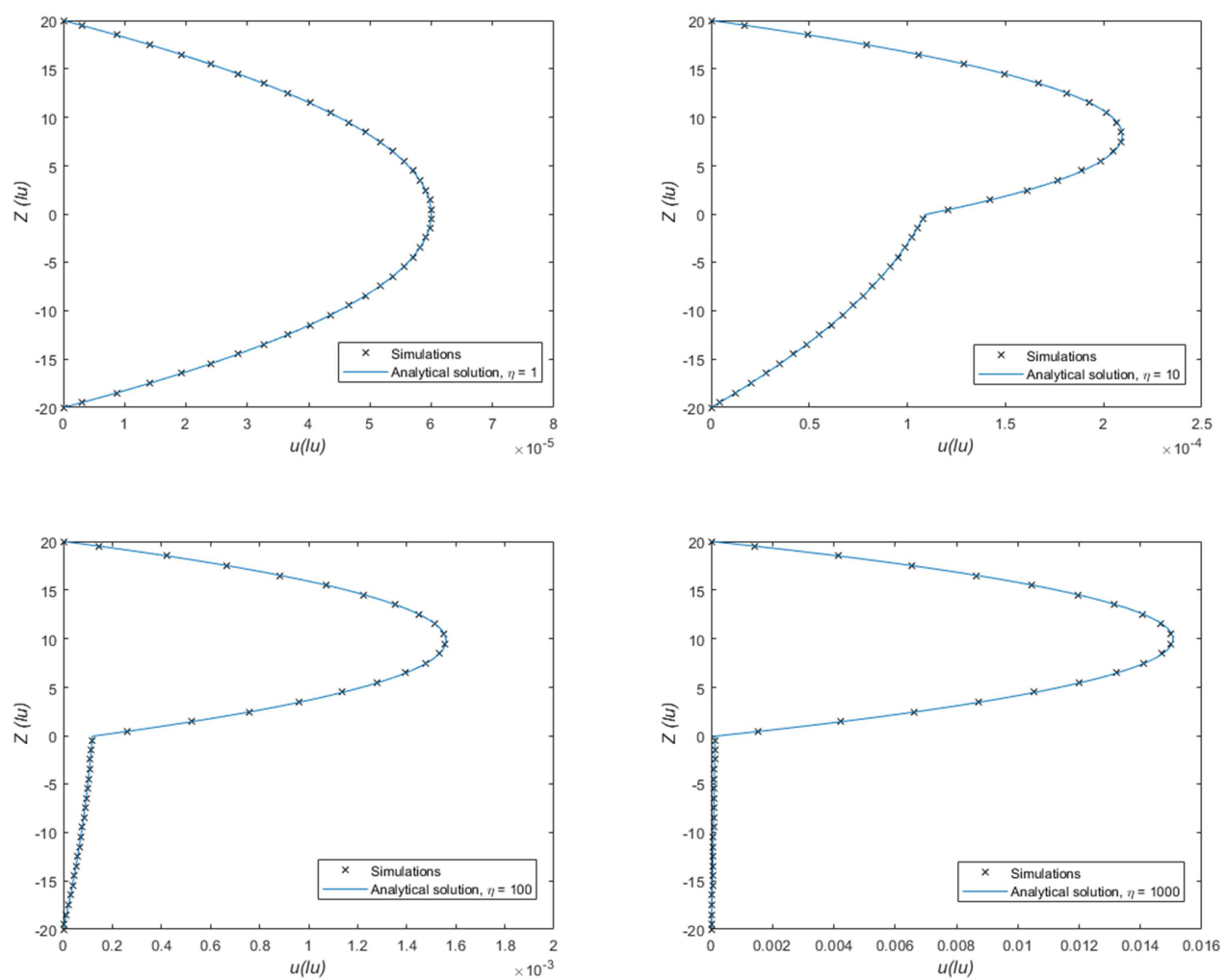

Fig. 3. Velocity profiles in two-phase layered flow with different viscosity ratio. 


\section{Contact angle}

Since the interaction of the contact line with the solid surface of the pore plays an important role in drop behavior, it is necessary to make sure that the wetting boundary conditions at the curved surface are implemented correctly. The following benchmark was considered: a drop was placed on a cylinder and let to equilibrate. The simulation domain was a 40×90×60 box. The no-slip simple halfway bounce-back wall conditions were employed for curved walls and the top and bottom faces in the $z$-direction, and periodic boundary conditions for the rest of the boundaries. The radius of the drop $(r)$ was $16 \mathrm{lu}$ and the radius of the cylinder $(R s)$ was 50 lu. The interface thickness was 6 lu. The initial state of the system in the $x=20$ lu plane is shown in Fig. 4(a). The specified and simulated static contact angles between the drop and the curved wall are shown in Fig. 4(b). The simulated angles agree well with the specified angles because maximum deviation is less than $2 \%$.

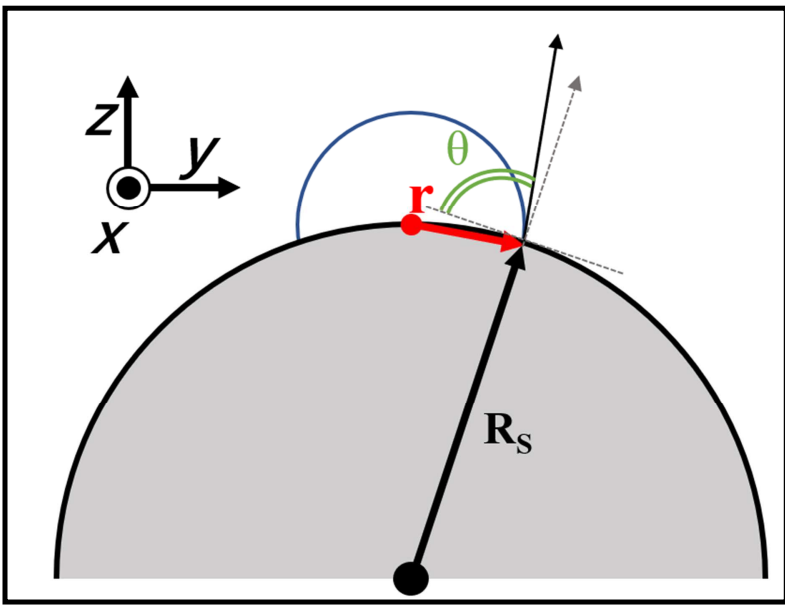

(a)

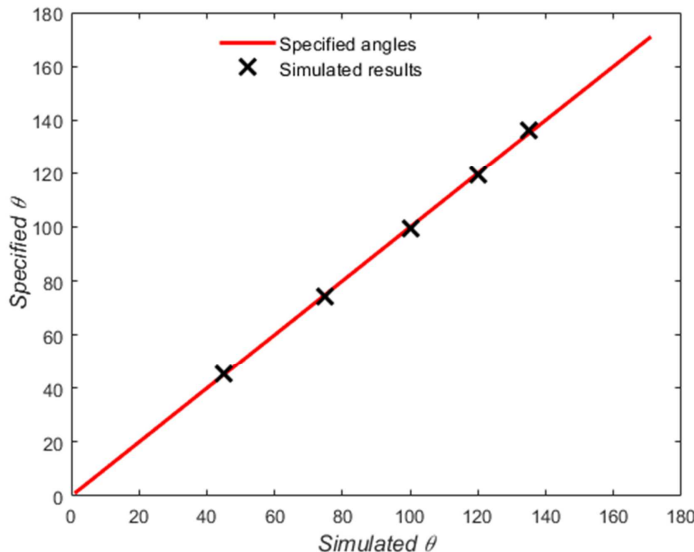

(b)

Fig. 4 (a) Schematic of the contact angle between a drop and a curved wall in the $x=20$ lu plane (b) Simulated and specified contact angles

\section{Mesh resolution}

Mesh sensitivity analyses were performed using a benchmark of gravity-driven drop motion in ambient surrounding liquid in a channel. For drop diameters $D=24,32$, and $40 \mathrm{lu}$, the terminal velocity was predicted. The simulation domain is shown in Fig. 5(a). The density ratio between dispersed and continuous liquids was $\rho_{\mathrm{d}} / \rho_{\mathrm{c}}=1.5$ and the liquids had matching 
viscosities. The interface thickness $(\xi)$ was $6 \mathrm{lu}$ and the surface tension $(\sigma)$ was $0.01 \mathrm{lu}$. Periodic boundary conditions were employed for the top and bottom faces and the no-slip bounce-back wall conditions for the rest of the boundaries. In Fig. 5(b), we present the non-dimensional drop velocity as a function of time for different drop diameters. Time and velocity are non-dimensionalized as $\bar{t}=t / \sqrt{D / g}$ and $\bar{u}=u / \sqrt{g D}$, respectively. The gravitational acceleration is set to $-2.37 \times 10^{-5},-1.0 \times 10^{-5,}$ and $-5.12 \times 10^{-6}$ in lu to achieve the same Reynold number. for $D=24,32$, and 40 lu, respectively. As we can see from Fig. 5(b), the drop velocity profiles almost overlap when the drop diameter is larger than $32 \mathrm{lu}$. It indicates that representing a drop diameter with $32 \mathrm{lu}$ is sufficient resolution.

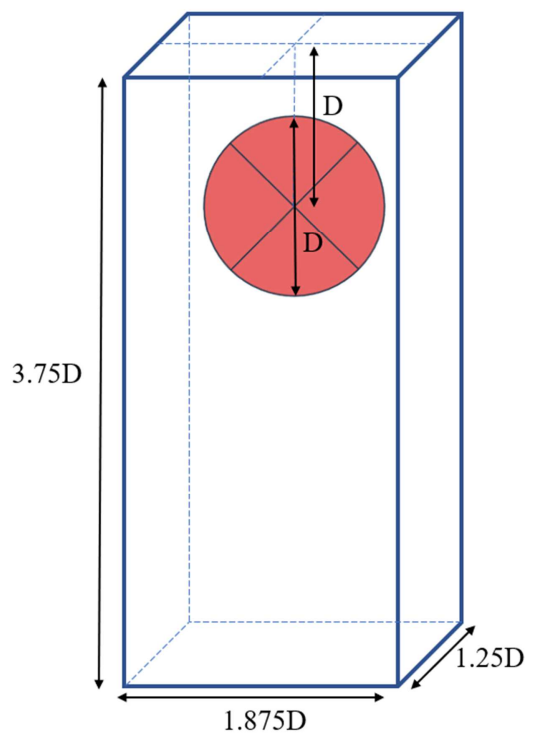

(a)

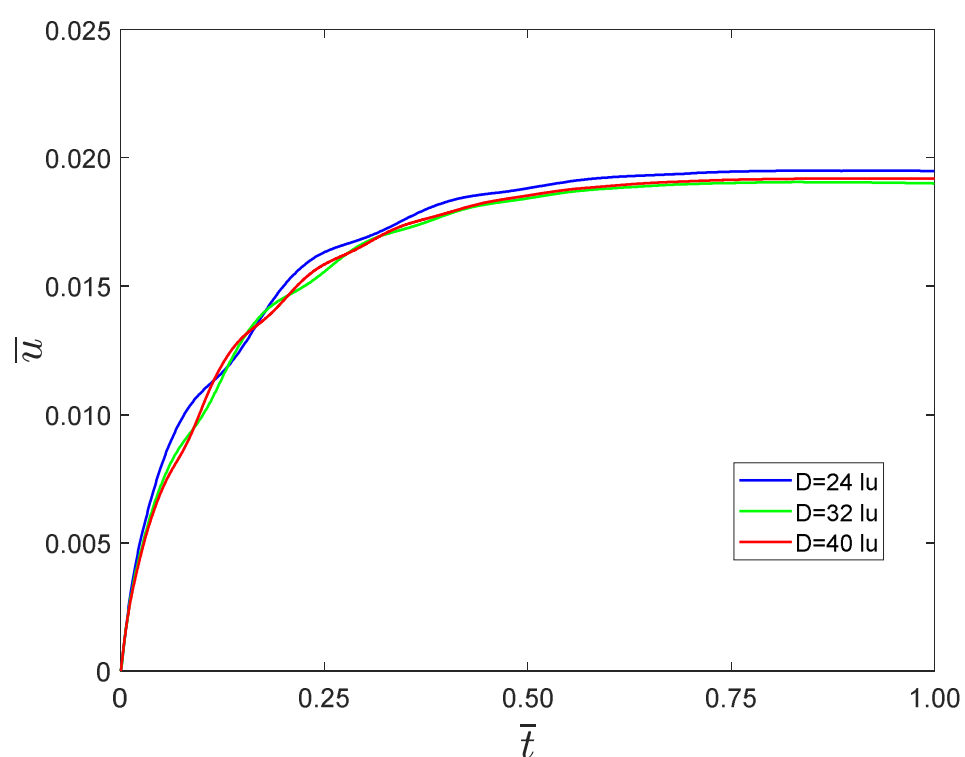

(b)

Fig. 5 (a) Simulation settings for the dense drop in the continuous phase (b) Non-dimensional drop velocity as a function of time for three mesh resolutions

\section{Results and discussion}

The results are presented in two parts. First, we consider a drop falling through a pore due to gravity in the ambient continuous liquid phase and compare our results to the experimental data of Ansari et al. (2018, 2019). This comparison is done for the purpose of qualitatively validating our model. In addition, we verify the prediction of the pressure change across the drop interface as it passes through the pore. In the second part, we present the results of drop breakup in the pore space when the carrier (surrounding) liquid is in continuous flow. The influence of governing dimensionless numbers (the 
Weber number, the Reynolds number, and the viscosity ratio) on drop deformation and breakup is investigated. A chart outlining the conditions for drop breakup is presented at the end.

\subsection{A drop falling through a single pore space due to gravity}

Ansari et al. (2018, 2019) carried out an experimental study of a Glycerol drop falling in ambient Canola oil through a pore using micro particle shadow velocimetry ( $\mu$-PSV). The simulation is set up to replicate the experiment in a domain of $40 \times 60 \times 180 \mathrm{lu}$. The droplet diameter $D$ is $32 \mathrm{lu}$. The simulated system matches experiment by the Eötvös number $\left(E o=\frac{g \Delta \rho D^{2}}{\sigma}=0.267\right)$ and Morton number $\left(M o=\frac{g \Delta \rho v_{c}^{4} \rho_{c}^{2}}{\sigma^{3}}=254\right.$ where $v_{\mathrm{c}}$ and $\rho_{\mathrm{c}}$ are the kinematic viscosity and density of continuous phase, respectively). The measured velocity of the drop was of the order of $10^{-5} \mathrm{~m} / \mathrm{s}$ and it took more than 160 seconds for the drop to pass through the pore space. If we match experimental conditions and replicate their results using our explicit scheme, the time step needs to be $1.04 \times 10^{-5} \mathrm{~s}$. It will require approximately $15,000,000$ time steps which is equivalent to 38,500 hours of calculation time to complete the simulation by 15 cores (2.5 GHz). To accelerate the process, we increased the body force that represents the effect of gravity by a factor of six and qualitatively compared our results with their findings. The three-phase contact angle at the curved wall is set to $180^{\circ}$, and we use the static contact angle which we introduced in Section 2 to handle this problem. The comparison of the drop shape at different time instances is shown in Fig. 6. Overall, the results are in good agreement. Minor deviation in drop shape can be due to several reasons. The complexity of the analysis of experimental data to recover drop shape (see the original works of Ansari et al. (2019)) affects the drop shape. The properties of the liquids (viscosity, density, and interfacial tension) were not measured in the experimental study for the considered liquids and were taken from reference data. As a result, there might be a slight discrepancy between the experimental settings and our settings that were based on the reference values of liquid properties. 


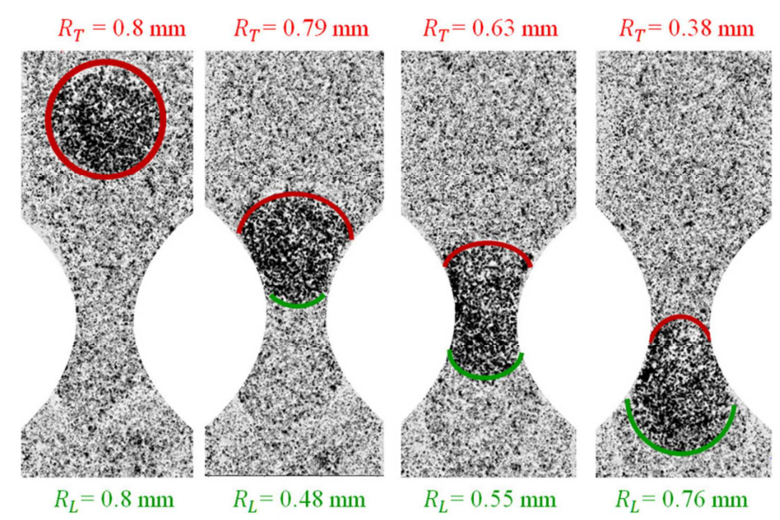

(a)

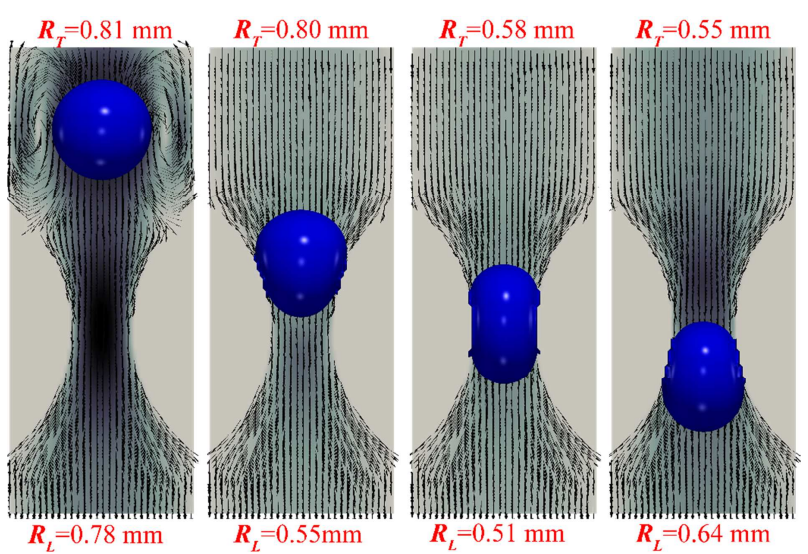

(b)

Fig. 6. (a) Experimental and (b) numerical results of gravity-driven motion of a drop through the pore space. $R_{T}$ and $R_{L}$ denote radii of trailing and leading ends of the drop, respectively

To verify our method quantitatively, we performed simulations of a similar problem with the goal to check the capability of the method to predict the pressure distribution inside the deforming drop and correlate it with the drop shape. The flow configuration is shown in Fig. 7: a drop moves along with the continuous phase through the pore. The dimensions of the channel are $40 \times 120 \times 270$ lu and the diameter of the cylindrical obstruction on the pore is $D_{l}=50 \mathrm{lu}$. The density and viscosity of the drop match the corresponding properties of the continuous phase: $\rho=1.0 \mathrm{lu}, \mu=1 / 3 \mathrm{lu}$. The droplet diameter is $32 \mathrm{lu}$, the interface thickness is $6 \mathrm{lu}$ and the surface tension is $\sigma=0.12 \mathrm{lu}$. The periodic boundary conditions are set at the top and bottom faces of the domain and the no-slip boundary conditions are set for the rest of the boundaries. The three-phase contact angle at the curved wall is set to $180^{\circ}$, meaning the drop is non-wetting. 
The simulation run was performed as follows. First, the drop of diameter $D=32$ lu was injected upstream the pore area and let to equilibrate during 1200 time steps. There was no body force applied at this stage. The final equilibrium shape of the drop was used as an initial condition for the second stage of the simulations when a body force of $\rho g=-2 \cdot 10^{-5}$ lu in $z$-direction was applied to the entire domain. During the run, the radii of the leading and the trailing ends of the drop were measured using a curve-fitting procedure. The details of notation are shown in Fig. 8. The droplet surface was identified at $\phi=0.5$. A Matlab code for curve fitting procedure was written to measure the radii of the leading and trailing faces in the $x=20$ lu and $y=60$ lu planes.

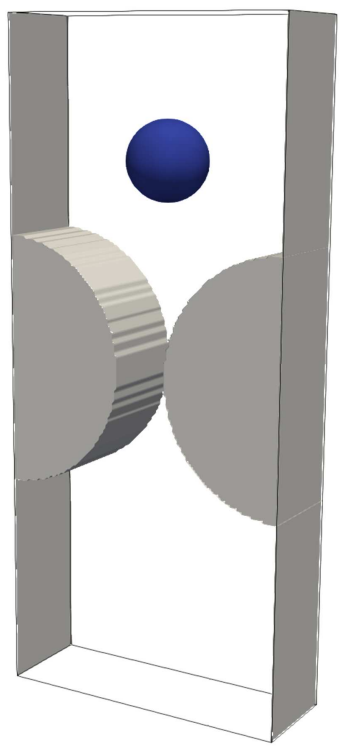

(a)

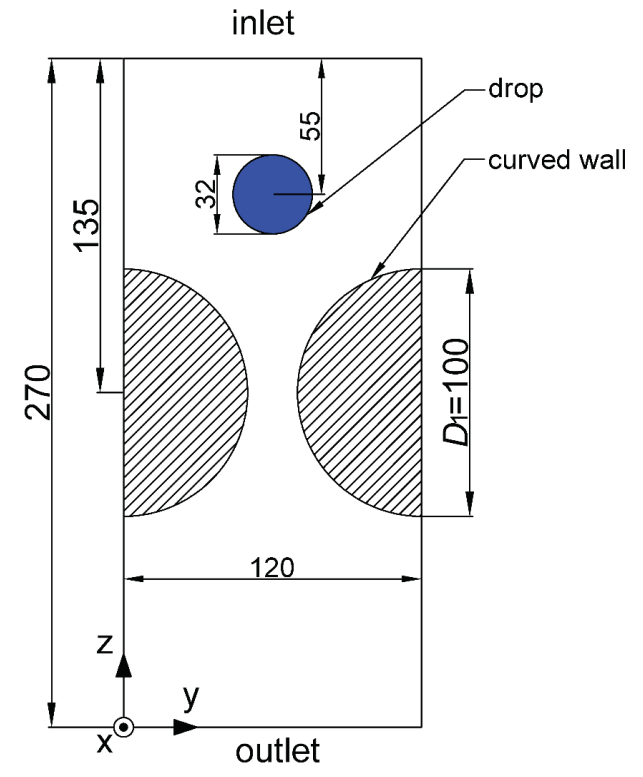

(b)

Fig. 7. (a) 3D Computational domain $40 \times 120 \times 270$ lu. (b) Cross-section of the domain at $x=20$ plane, dimensions in lu.
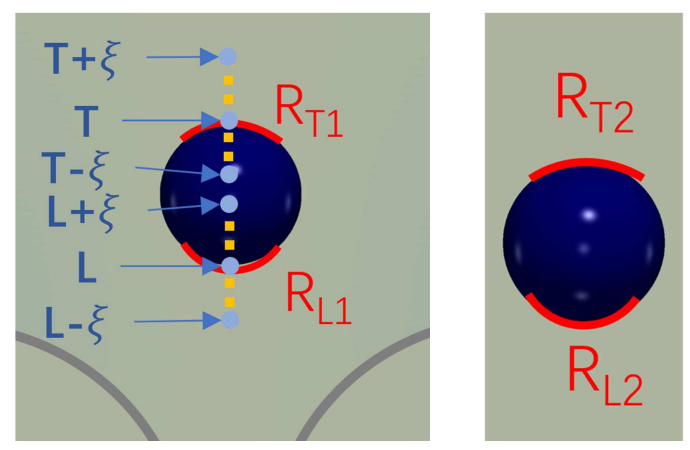

Fig. 8. The notation to determine the radii of the leading and the trailing ends of the drop in (y-z) (left) and (x-z) (right) 
Once the body force is turned on, the drop starts moving and deforming downstream the channel (Fig. 9). At $\bar{t}=13.0$, the drop approaches the upper part of the pore. The leading end of the drop is squeezed. The center of mass of the drop reaches the center of the pore at $\bar{t}=17.4$. The drop is stretched in the z-direction, and the leading and trailing ends are almost symmetrical. The drop leaves the pore area at $\bar{t}=21.8$. Due to the expansion of the flow after the pore, the velocity of the leading end is significantly smaller than the velocity of the trailing end. This explains the change in the drop shape: the drop is squeezed in the $z$-direction. Finally, the drop restores the spherical shape and moves down the channel.

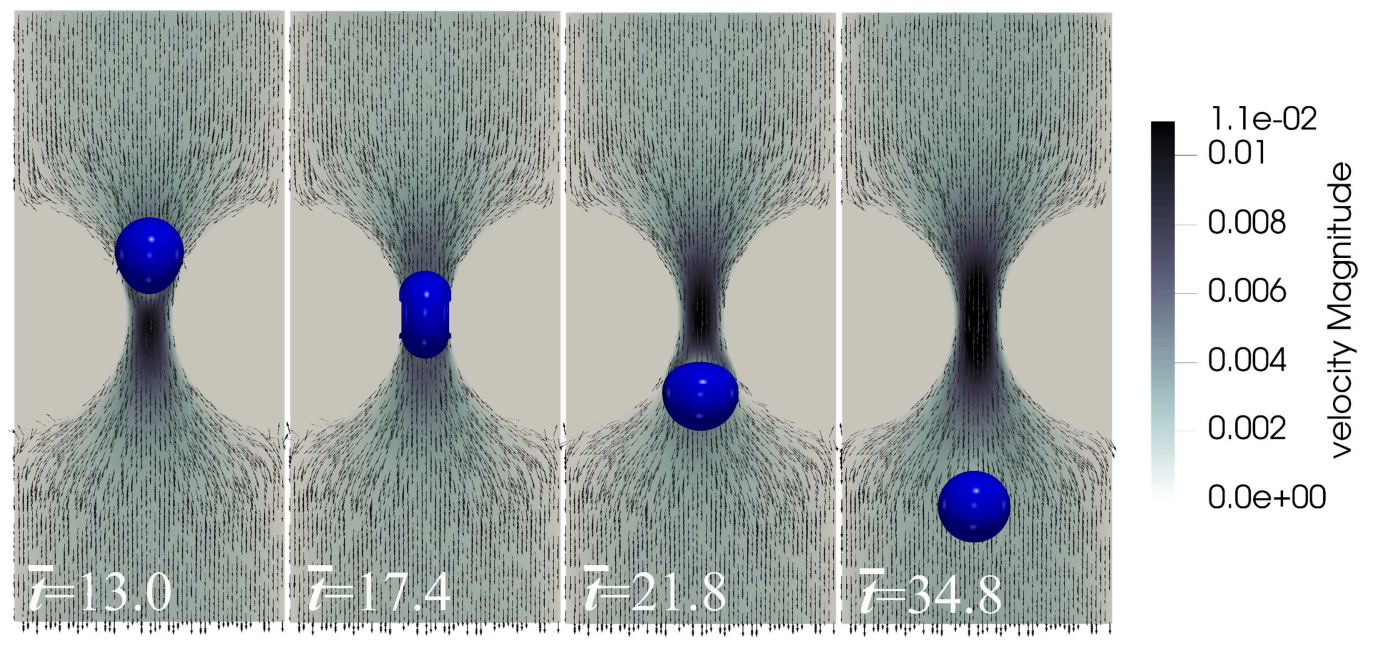

Fig. 9. A drop passing through a pore waist in the $x=20$ lu plane

The main purpose of this verification case is to assess the correctness of the pressure field obtained numerically in case of drop motion within a complex geometry in the presence of the body force.

The estimated pressure difference between the leading and trailing ends of the drop due to the surface tension is given by:

$$
\Delta P_{e s t-L T}=\Delta P_{e s t-L}-\Delta P_{e s t-T}=\sigma\left(\frac{1}{R_{L 1}}+\frac{1}{R_{L 2}}\right)-\sigma\left(\frac{1}{R_{T 1}}+\frac{1}{R_{T 2}}\right)
$$

The pressure difference between the leading end $\left(\Delta P_{L B M-L}\right)$ and trailing end $\left(-\Delta P_{L B M-T}\right)$ due to the surface tension can also be calculated by the pressure fields in the LBM simulations minus the effects of the velocity fields and $\boldsymbol{F}_{\mathbf{b}}$ as follows:

$$
\Delta P_{L B M-L}=P_{(\mathrm{L}+\xi)}-\mathrm{P}_{(\mathrm{L}-\xi)}-2 \xi\left\{-\rho \frac{\partial u_{z}}{\partial t}+\mu \frac{\partial^{2} u_{2}}{\partial z^{2}}+F_{b z}\right\}
$$




$$
\begin{gathered}
\Delta P_{L B M-T}=P_{(\mathrm{T}-\xi)}-\mathrm{P}_{(\mathrm{T}+\xi)}+2 \xi\left\{-\rho \frac{\partial u_{z}}{\partial t}+\mu \frac{\partial^{2} u_{2}}{\partial z^{2}}+F_{b z}\right\} \\
\Delta P_{L B M-L T}=\Delta P_{L B M-L}-\Delta P_{L B M-T}
\end{gathered}
$$

where $P_{(L+\xi)}$ and $P_{(L-\xi)}$ (See Fig. 8) are the pressure inside and outside the leading end of the drop, respectively; $P_{(\mathrm{T}-\xi)}$ and $\mathrm{P}_{(\mathrm{T}+\xi)}$ (See Fig. 8) are the pressure inside and outside the trailing face, respectively.

As shown in Fig. 10, the pressure differences between the leading and trailing ends of the drop estimated using Eq. (34) and predicted numerically are in good agreement. This means that the variations in the velocity and pressure fields reflect on the shape of the drop correctly.

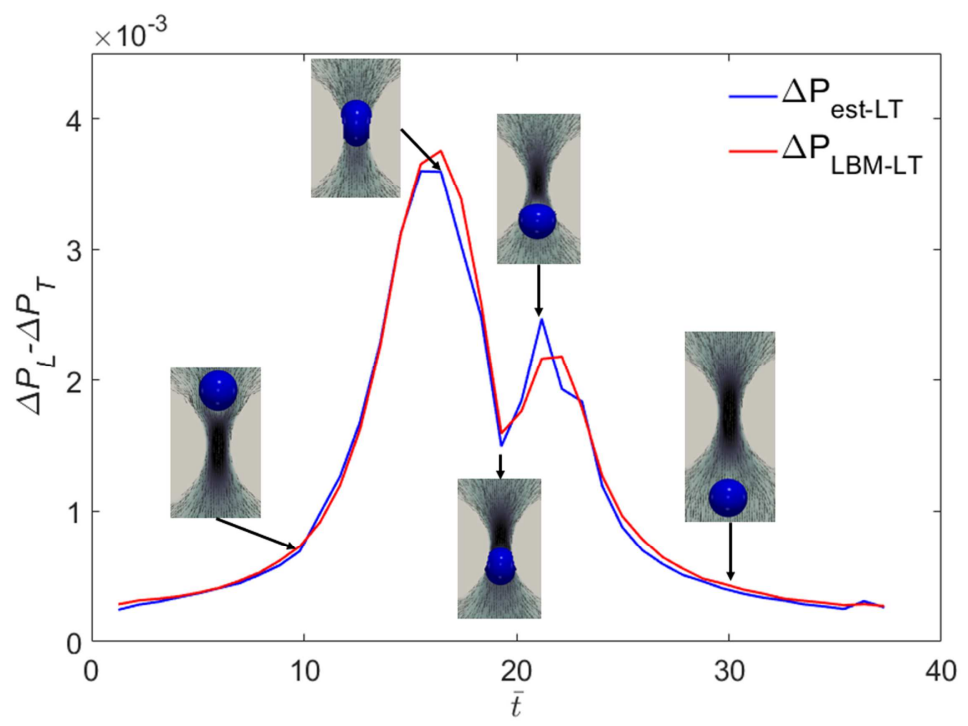

Fig. 10. The pressure difference between the leading and trailing ends of the drop as it passes through the pore $\left(\Delta P_{e s t-L T}\right.$ and $\Delta P_{L B M-L T}$ refer to Eq.(34) and Eq.(35c), respectively)

\subsection{Drop breakup in the pore space}

We used the same pore geometry as depicted in Fig. 7 to study the conditions of drop breakup. In all cases, the continuous phase was moving downward in the z-direction with the average velocity in the $z=270$ lu plane denoted as $u_{\text {avg }}$ This motion was created by applying a constant body force to the entire domain. For each case, the steady-state velocity field of a single-phase flow was obtained first. As an example, a velocity field in cross-section $y=60$ for $R e=0.26$ is shown in Fig. 11(a). The average velocity in straight sections of the channel (away from the pore space) is 0.0029 lu, 
and the maximum velocity at the pore throat is $0.034 \mathrm{lu}$. Such velocity fields were used as an initial condition for further two-phase flow simulations.

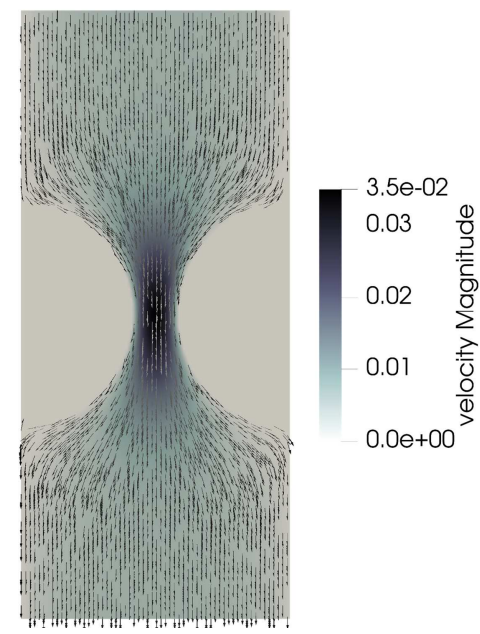

(a)

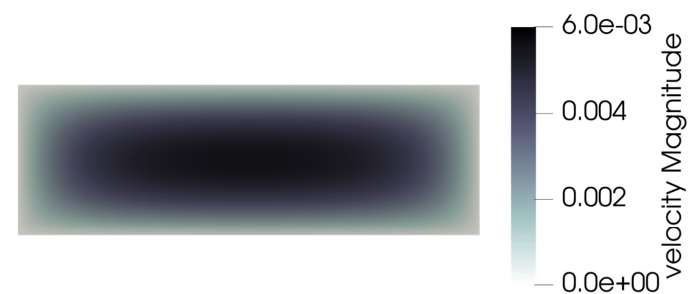

(b)

Fig. 11. (a) Velocity field of the single-phase flow in $x=20$ lu plane (b) velocity field at the inlet ( $z=270$ lu plane)

The rest of the simulation parameters are as follows. The densities of the drop and the continuous phase are the same: $\rho=1.0 \mathrm{lu}$. The contact angle was set to $180^{\circ}$, the thickness of the interface was $6 \mathrm{lu}$, the mobility $M=0.2$ (Mitchell et al., 2018).

Three forces define the behavior of the drop motion: surface tension, inertial, and viscous forces. The following set of dimensionless numbers can be used to describe the relative effect of these forces: the Reynolds number $R e$ (inertial vs viscous force), Weber number We (inertial vs surface tension force), the capillary number $C a$ (viscous to surface tension force), and the viscosity ratio $\eta$ :

$$
R e=\frac{\rho_{c} u_{\mathrm{avg}} R_{h}}{\mu_{c}} \quad W e=\frac{\rho_{c} u_{\mathrm{avg}}^{2} R_{h}}{\sigma} \quad C a=\frac{\mu_{\mathrm{c}} u_{\mathrm{avg}}}{\sigma} \quad \eta=\frac{\mu_{\mathrm{d}}}{\mu_{\mathrm{c}}}
$$

where $\rho_{\mathrm{c}}$ is the density of continuous phase, $u_{\mathrm{avg}}$ is the average velocity of the single-phase flow of continuous liquid, $R_{\mathrm{h}}$ is the hydraulic radius of the channel defined as $\frac{40 \times 120}{40+120}=30 \mathrm{lu}, \sigma$ is the interfacial tension between the liquids, $\mu_{\mathrm{c}}$ is the dynamic viscosity of the continuous phase and $\mu_{\mathrm{d}}$ is the dynamic viscosity of the drop. For each case, we also estimated the Ohnesorge number

$$
O h=\frac{\sqrt{W e}}{R e}=\frac{\mu_{c}}{\sqrt{\rho_{c} \sigma R_{h}}}
$$


We conducted a series of simulations to investigate the influence of the surface tension (the Weber number), the average velocity of the continuous flow (the Reynolds number), and the viscosity ratio $(\eta)$ on drop deformation and breakup. The flow parameters for each case are shown in Table 1. The simulation cases were chosen as follows. Case 2 represents a baseline case: matching densities of liquids with $\rho=1$, the viscosity of continuous phase $\mu_{\mathrm{c}}=1 / 3$ and viscosity ratio $\eta=1$ such that the corresponding relaxation time $\tau_{f}=1.0$ everywhere in the domain, and the surface tension $\sigma=0.005$. Cases 1,3 , and 4 retain parameters of Case 2 except for surface tension, therefore Cases 1-4 show the effect of surface tension (fixed $R e$ and varying $\mathrm{We}$ ). Cases 5 and 6 have baseline parameters except for the viscosity of liquids, i.e. Cases 2, 5, and 6 provide data to explore the effect of $R e$ at fixed $W e$. Cases 7 and 8 retain the parameters of Case 2 except for the viscosity ratio. Mesh sensitivity analysis of Case 1 is shown in Appendix D.

Table 1. Simulation cases and drop breakup output

\begin{tabular}{cccccc|cc|c}
\hline Case & $R e$ & $W e$ & $C a$ & $O h$ & $\eta$ & $\mu_{\mathrm{c}}$ & $\sigma$ & Drop breakup \\
\hline 1 & 0.26 & 0.03 & 0.10 & 0.61 & 1.00 & $1 / 3$ & 0.01 & no \\
2 & 0.26 & 0.05 & 0.19 & 0.86 & 1.00 & $1 / 3$ & 0.005 & yes \\
3 & 0.26 & 0.10 & 0.39 & 1.22 & 1.00 & $1 / 3$ & 0.0025 & yes \\
4 & 0.26 & 0.25 & 0.97 & 1.92 & 1.00 & $1 / 3$ & 0.001 & yes \\
5 & 0.52 & 0.05 & 0.10 & 0.43 & 1.00 & $1 / 6$ & 0.005 & no \\
6 & 2.61 & 0.05 & 0.02 & 0.09 & 1.00 & $1 / 30$ & 0.005 & no \\
7 & 0.26 & 0.050 & 0.19 & 0.86 & 0.10 & $1 / 3$ & 0.005 & yes \\
8 & 0.26 & 0.050 & 0.19 & 0.86 & 0.01 & $1 / 3$ & 0.005 & yes \\
\hline
\end{tabular}

\subsubsection{The effect of the surface tension}

Surface tension plays a significant role in the multi-phase flow through porous media. In this series of simulations, the Reynolds number is fixed to $R e=0.26$, the viscosity ratio $\eta$ is equal to 1.0 and we change the value of the surface tension that results in a change of the Weber number (Cases 1-4). As shown in Figure 12, the mesh with $\mathrm{D}=32$ lu has reached mesh independence because the results have good agreement with $\mathrm{D}=48 \mathrm{lu}$. The drop diameter is $D=32 \mathrm{lu}$. The shapes of 
the drops at different time instances are shown in Fig. 12 for four cases. The time was non-dimensionalized as $\bar{t}=$ $t / \sqrt{D / g}$

An equilibrated drop is injected at $\bar{t}=0$ into the steady-state flow of continuous liquid. In the case with We $=0.03$ (highest surface tension) the drop does not break (Fig. 12 (a)). When the surface tension is decreased (We=0.05), the drop breaks producing two satellites (Fig. 12(b)). The higher velocity values of the continuous phase at the centerline in the $y=60$ plane (pore throat) compared to the channel flow create a dent in the trailing end of the drop at $\bar{t}=8.75$ (see Fig. 12 (b)). The trailing end of the drop forms two long threads at $\bar{t}=11.50$. Then a neck forms. The neck thins gradually, and the drop breaks at $\bar{t}=13.00$ because of end pinching.

With the decrease of the surface tension (an increase of the Weber number), the threads formed behind the trailing end of the drop split into more satellite and sub-satellite fragments. In Case 3 (see Fig. 12 (c)), the two sub-satellite coalesce with two satellite drops at $\bar{t}=15.50$. The satellite drops decelerate due to the expansion of the flow so that the subsatellites can catch up and coalesce. Finally, the drops generate four satellites when it passes through the cylinder pore space, as shown in Fig. 12 (c).

In summary, higher surface tension (lower Weber number) prevents drop breakup. For the fixed $R e=0.26$, there is no breakup at $W e=0.03$, and the drops break up into 3,5,5 fragments when they leave the pore space with higher Weber number. We conclude that drops with lower values of surface tension (high $\mathrm{We}$ ) break more easily producing multiple fragments. The coalescence of fragments is also observed during this process due to the special structure of the (flow through the) cylinder pore. 


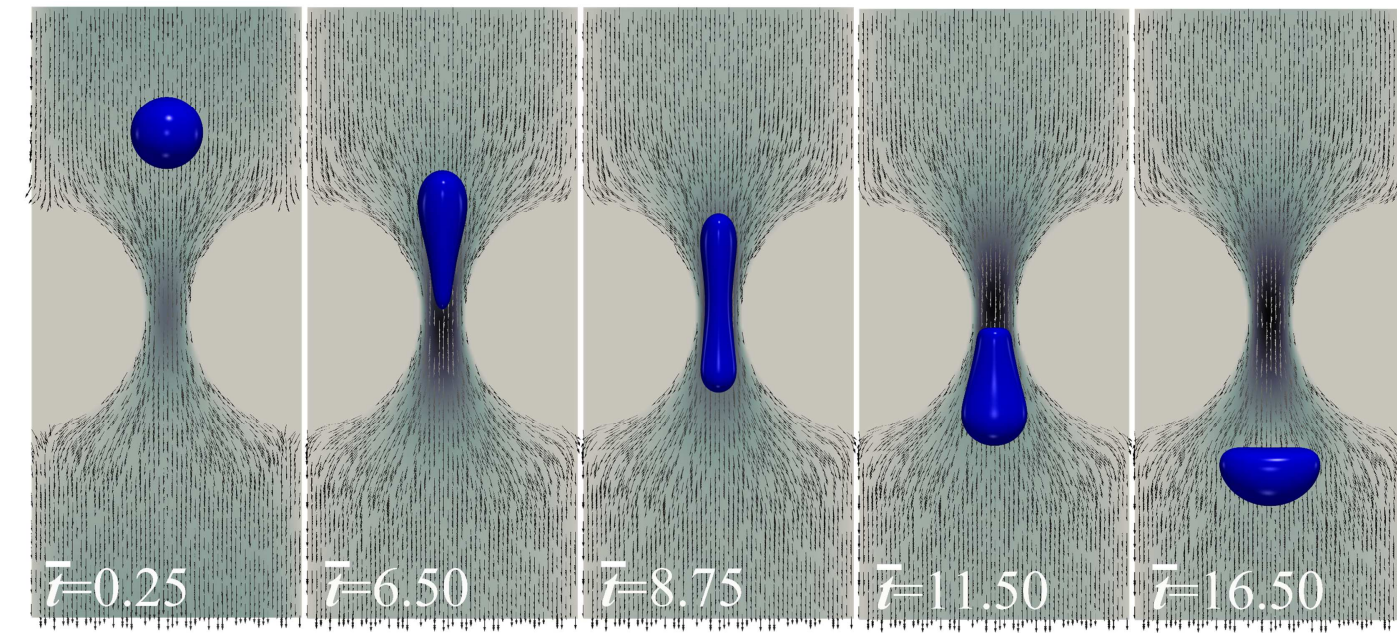

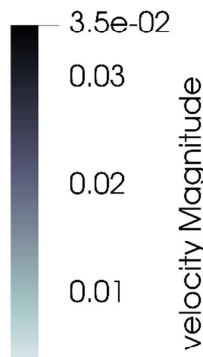

$0.0 e+00$

(a)

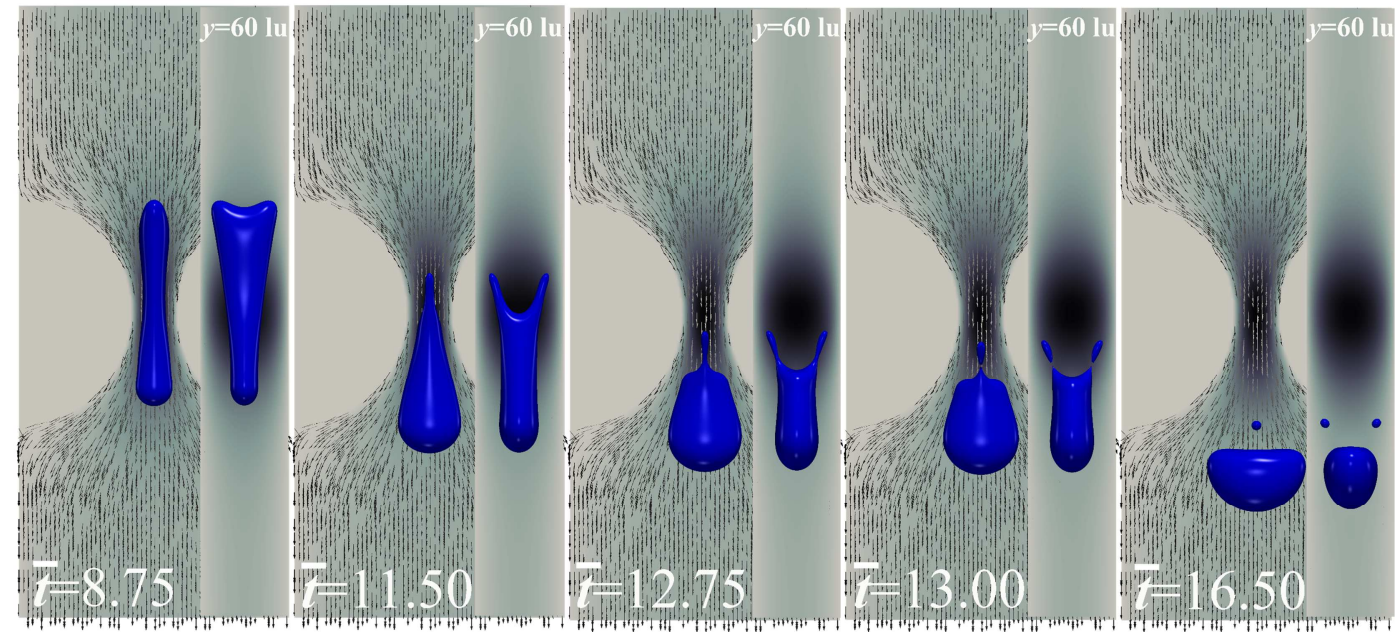

(b)

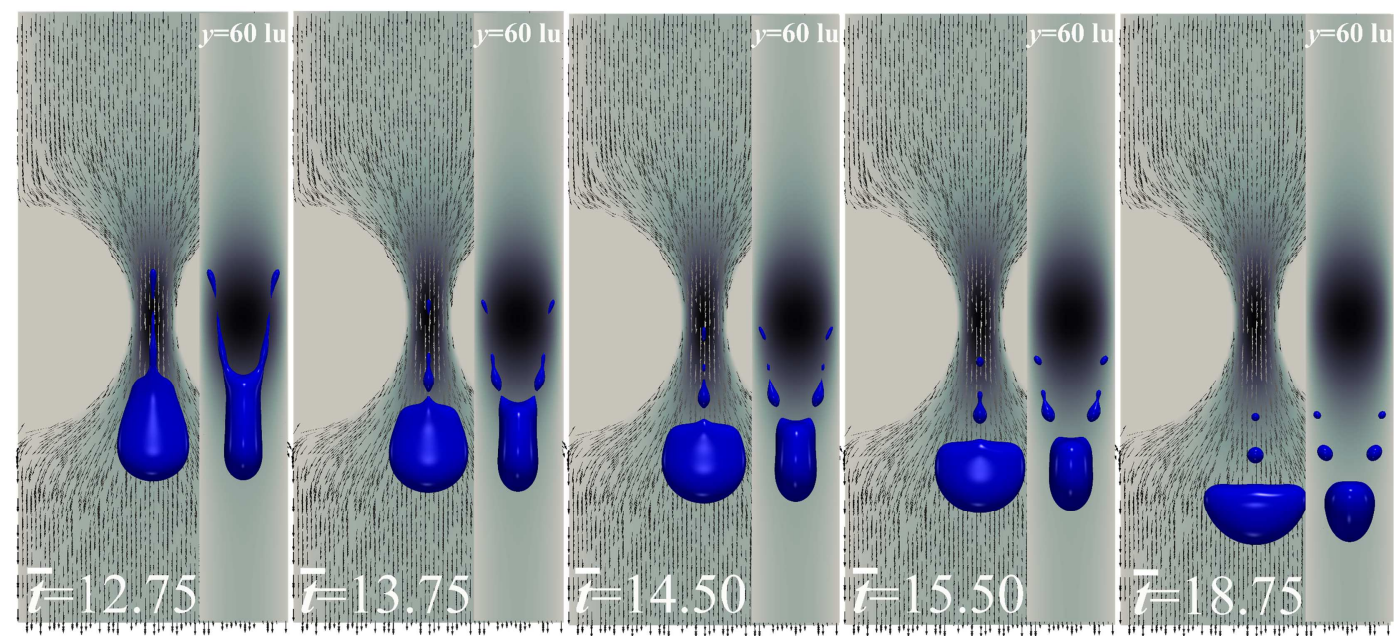

(c) 


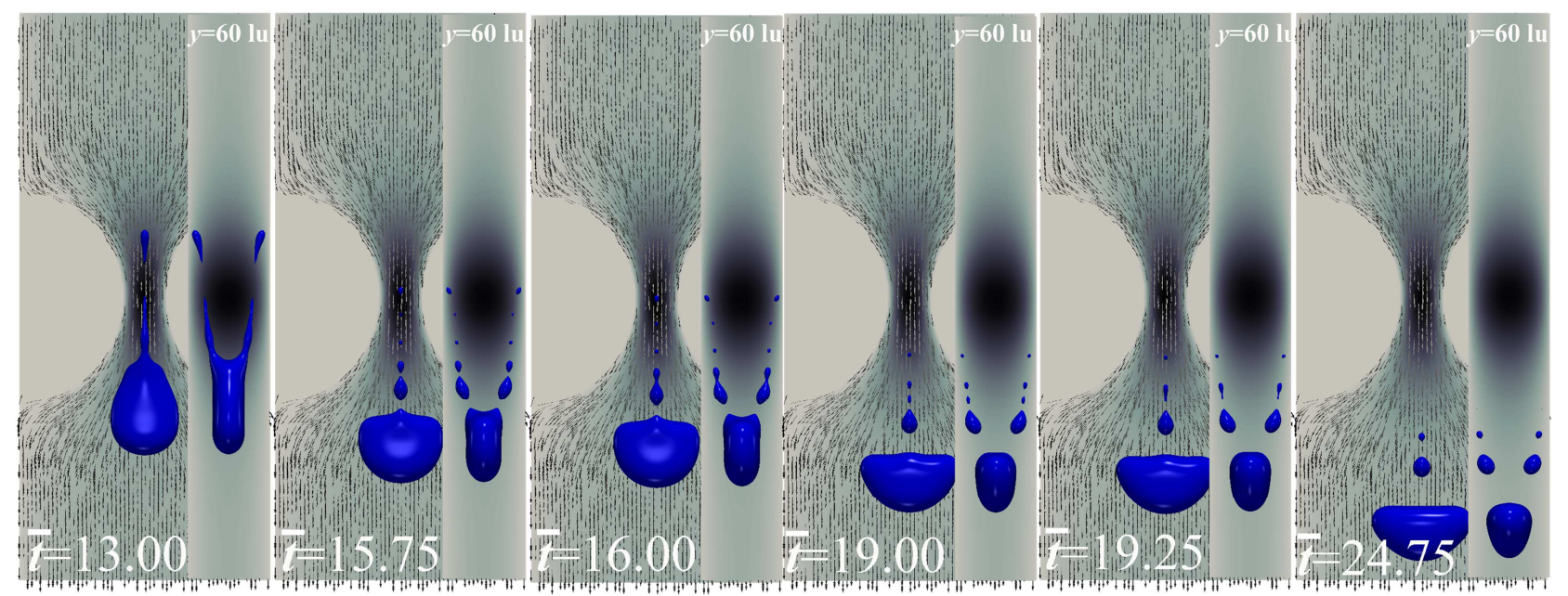

(d)

Fig. 12 Effect of Weber number on drop deformation and breakup. The process of drop motion through a pore with at $R e=0.29$ and (a) $W e=0.03$ in the $x=20$ lu plane (b) $W e=0.05$, (c) $W e=0.10$ and (c) $W e=0.25$ in the $x=20$ lu and $y=60$ lu plane.

\subsubsection{The effect of $R e$}

In this set of simulations, the Weber number is fixed to $W e=0.05$ and the Reynolds number is varied by changing the viscosity of the continuous liquid (Cases 2,5 , and 6 representing $R e=0.26,0.52$ and 2.61 , respectively). The viscosity ratio is set to unity. The drop shape at different time instances for Cases 5 and 6 are shown in Fig. 13 (Case 2 is presented in Fig. 12 (b)). The drop does not break in any of the considered cases.

To quantify the deformation of the drops as they move through the pore in these three cases, two deformation parameters $D^{*}$ and $L^{*}$ following the work by Olgac et al. (2006) are introduced. $D^{*}$ is defined as the ratio of the perimeter of the deformed drop profile to that of the equivalent spherical drop in the $x=20$ plane, and $L^{*}$ as the axial length of drop profile scaled by the height of the pore (see $D_{1}$ in Fig. 4(b)). The deformation of the drops in these three cases represented by $D^{*}$ and $L^{*}$ are shown in Fig. 14 where $z^{*}$ is the nondimensional position of the drop center scaled by the height of the pore $D_{1}$ calculated by $\mathrm{z}^{*}=(\mathrm{z}-85) / D_{1}$, that is $\mathrm{z}^{*}=0$ for the drop at the bottom of the pore and $\mathrm{z}^{*}=1$ for the drop at the top of the pore. The $D^{*}$ and $L^{*}$ have the maximum value when the drop is at the pore center $\left(z^{*}=0.5\right)$. The deformation is very small for case $6(R e=2.61)$, and the largest deformation occurs in Case 2 at $R e=0.26$. The deformation decreases as the 
Reynolds number increases indicating that drop breakup probability decreases with the increase of the $R e$ at fixed Weber number.

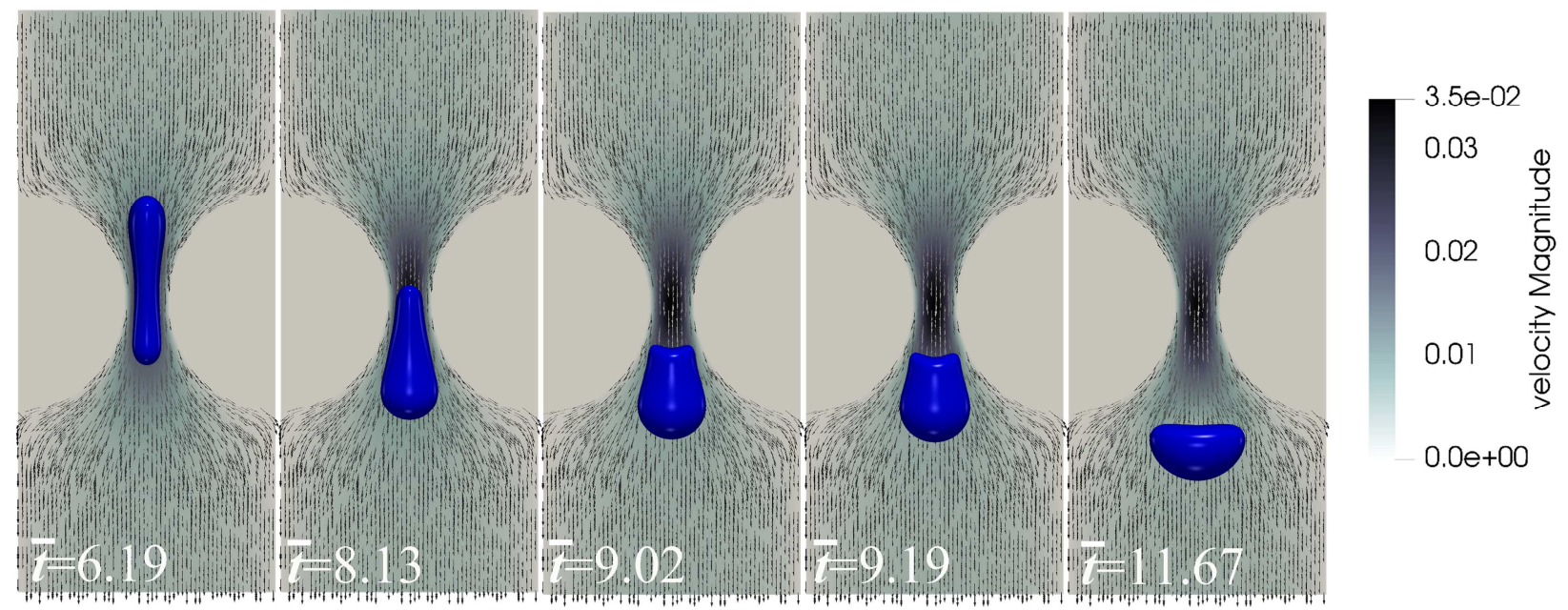

(a)

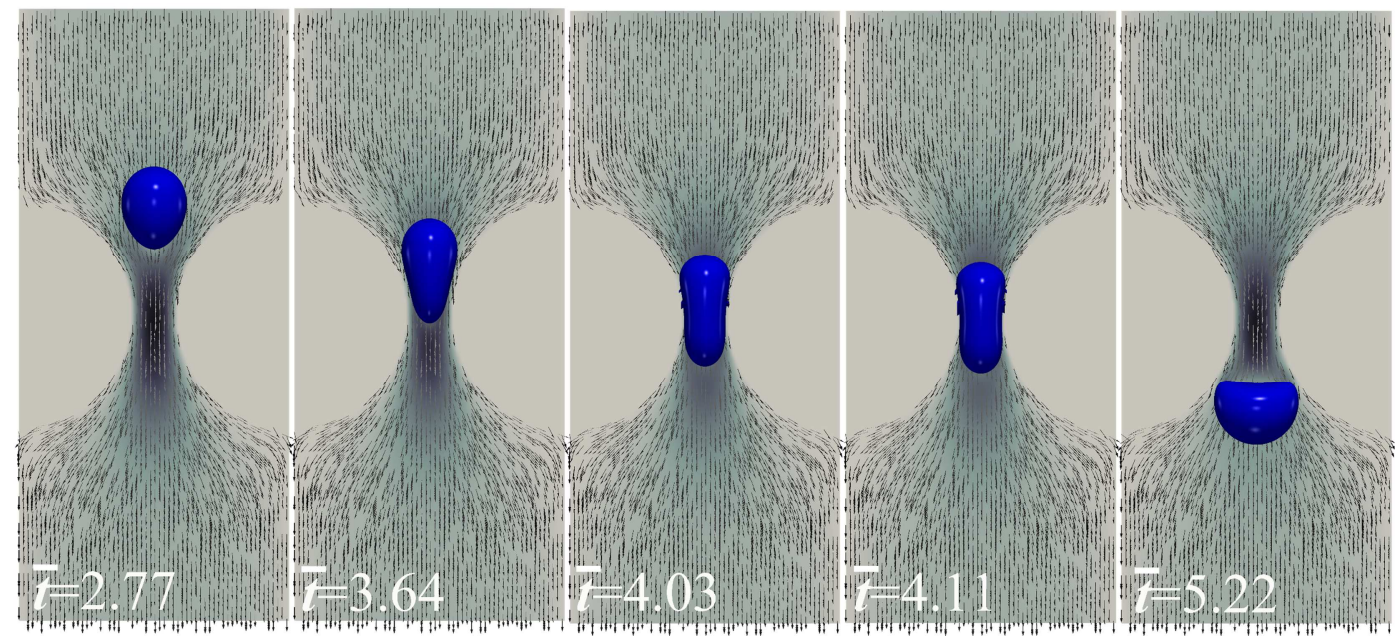

(b)

Fig. 13 The process of a drop motion through a pore at $W e=0.05$ and (a) $R e=0.52$ (b) $R e=2.61$ in the $x=20$ lu and $y=60$ lu plane
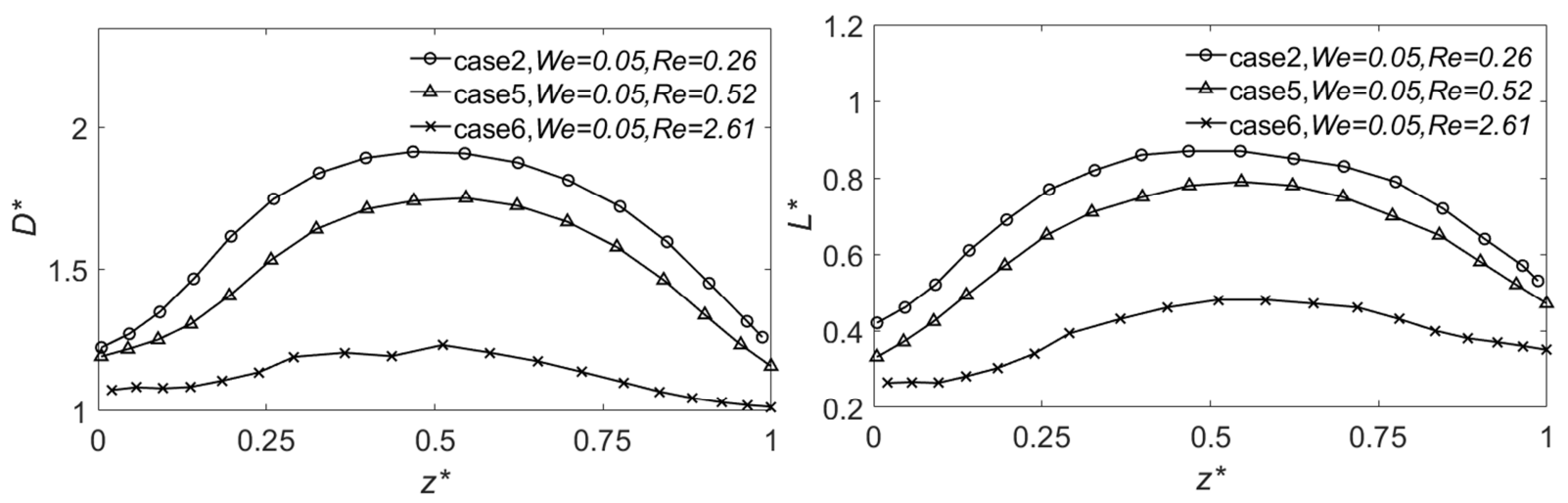
Fig. 14 The deformation parameters of the drops through a pore at $W e=0.05$ and $R e=0.26,0.52$ and 2.61.

\subsubsection{The effect of viscosity ratio}

The viscosity ratio $\eta$ is an important parameter to determine the drop breakup conditions (Zhao, 2007; Komrakova et al., 2014). Three viscosity ratios $(\eta=1,0.1$, and 0.01$)$ were chosen to study the effects on the breakup conditions (Cases 2 , 7, and 8). Other parameters are kept the same in these three cases $(R e=0.29, W e=0.05)$. The process of a drop motion through a pore with viscosity ratios equal to 0.1 and 0.01 is shown in Fig. 15 . The case with $\eta=1$ (Case 2 ) has been discussed in Fig. 12(b).

The process of the drop through the pore with $\eta=0.1$ is shown in Fig. 15(a). A neck deforms and thins gradually, and then the drop breaks at $\bar{t}=11.00$. There is a formation of two small satellite drops above the main body when the drop leaves the cylinder pore space at $\bar{t}=13.75$. The breakup of the case with $\eta=0.01$ occurs at $\bar{t}=10.75$ and the two generated satellite drops are the smallest when compared to those generated by $\eta=1$ and 0.1 .

To summarize, the higher values of drop viscosity promote drop breakup in the pore space. This observation is at odds with the research studies concerning drop deformation and breakup in a simple shear flow where viscous drops are more difficult to break (Zhao, 2007; Komrakova et al., 2014). We observe that drop breakage occurs at the two long threads, which are eroded and shaped by the high velocity of the continuous phase. Higher viscosity drops have a lower relative velocity with the continuous phase and long residence time when they go through the middle part of the pore. Long residence time give enough time to the continuous phase to erode the long threads. That is the reason why viscous drops can be broken more easily. 


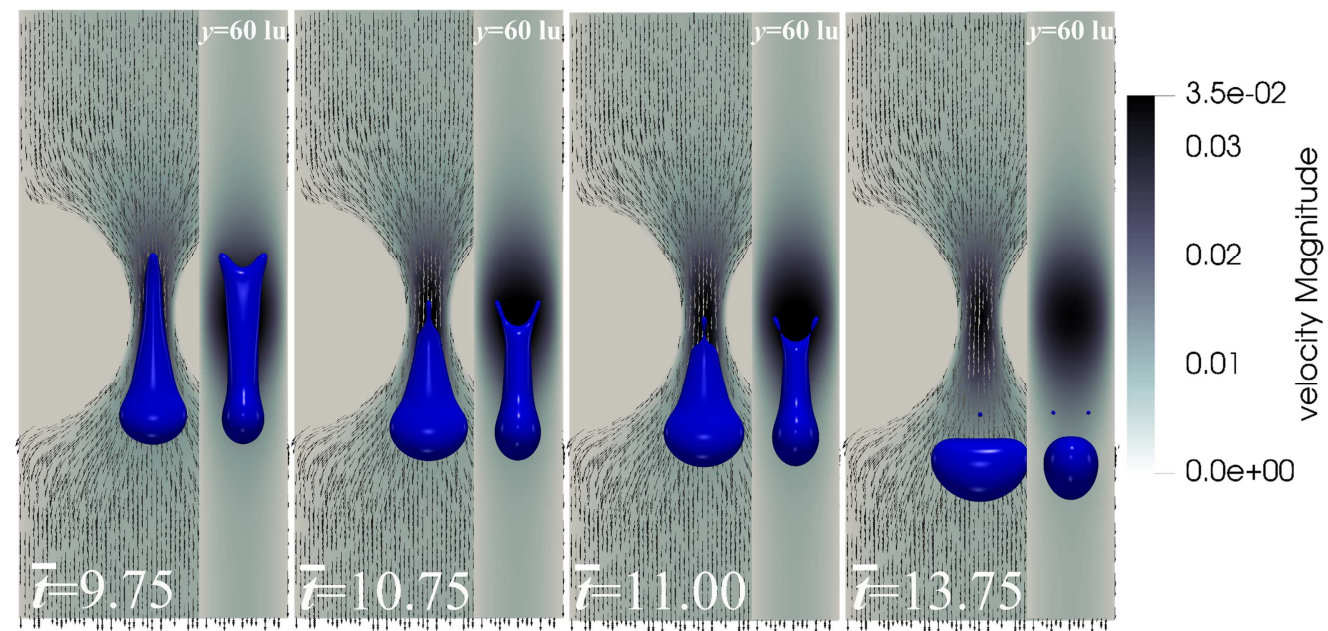

(a)

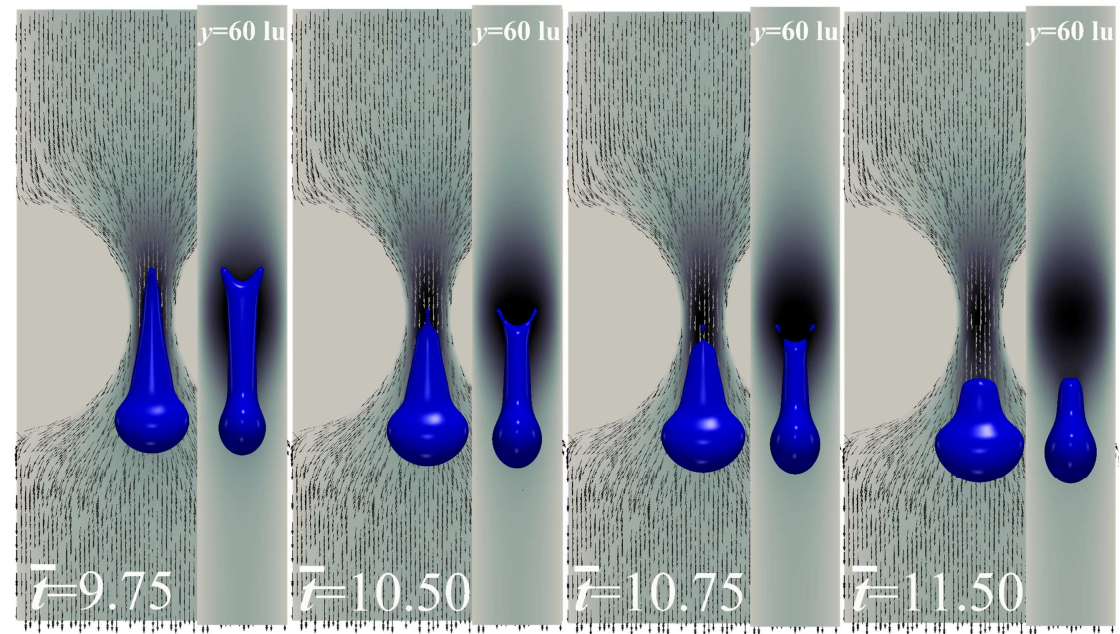

(b)

Fig. 15 (a) The process of a drop through a pore in the $x=20$ lu and $y=60$ lu plane at $R e=0.26$ and $W e=0.050$ with (a)

$$
\eta=0.1 \text { and (b) } \eta=0.01 \text {. }
$$

\subsubsection{Summary}

In previous sections, the influence of governing dimensionless numbers (the Weber number, the Reynolds number, and the viscosity ratio) on drop deformation and breakup were discussed. An additional 28 simulation cases were conducted to acquire more information to outline the drop breakup conditions. The definition of these cases is shown in Table 2 .

Fig. 16(a) shows the series of simulation results for $\eta=1$, along with the locations of these simulated results on a $C a$ versus We phase chart. The green constant $R e$ lines show that inertial forces dominate viscous forces in the bottom right 
corner of the chart while the viscous forces dominate inertial forces in the top left corner of the phase chart. From the phase chart, we can see that flow conditions from the bottom left (strong surface tensions) and bottom right (inertial forces dominate viscous forces) prevent drop breakup. In this force balance system, the lower surface tension and larger viscous forces that dominate inertial forces, are the key factors for the breakup. To find the critical breakup conditions, a We versus $R e$ map for $\eta=1$ is shown in Fig. 16(b). There is a clear dividing line between the breakup and no-breakup conditions, and the values of $W e$ on this line increase with $R e$. We use the breakup cases near the critical conditions to fit a dividing line in the dual-logarithm map. The relationship between $R e$ and $W e$ on this line is $W e=0.193 R e^{1.096}$. As shown in Fig. 17, the $C a$ versus $W e$ phase chart and $W e$ versus $R e$ map for $\eta=0.01$ almost have the same trend as for $\eta=1$. However, a lower surface tension is necessary to break the drop at $\eta=0.01$, the value of Weber number on the dividing line is $W e=0.216 R e^{1.104}$ in Fig. 17(b). Although these two dividing lines are very close, critical We is smaller for more viscous droplet $(\eta=1)$ to break up at constant $R e$. 
Table 2. All other cases for the summary section

\begin{tabular}{cccccc|cc|c}
\hline Case & $R e$ & $W e$ & $C a$ & $O h$ & $\eta$ & $\mu_{\mathrm{c}}$ & $\sigma$ & Drop breakup \\
\hline 9 & 0.26 & 0.046 & 0.18 & 0.82 & 1.00 & $1 / 3$ & 0.0055 & yes \\
10 & 0.26 & 0.042 & 0.16 & 0.79 & 1.00 & $1 / 3$ & 0.006 & no \\
11 & 0.26 & 0.034 & 0.13 & 0.70 & 1.00 & $1 / 3$ & 0.0075 & no \\
12 & 0.52 & 0.101 & 0.19 & 0.61 & 1.00 & $1 / 6$ & 0.0025 & yes \\
13 & 0.52 & 0.090 & 0.17 & 0.58 & 1.00 & $1 / 6$ & 0.0028 & yes \\
14 & 0.52 & 0.084 & 0.16 & 0.56 & 1.00 & $1 / 6$ & 0.003 & no \\
15 & 2.61 & 0.505 & 0.19 & 0.27 & 1.00 & $1 / 30$ & 0.0005 & no \\
16 & 2.61 & 0.561 & 0.21 & 0.29 & 1.00 & $1 / 30$ & 0.00045 & yes \\
17 & 2.61 & 0.025 & 0.01 & 0.06 & 1.00 & $1 / 30$ & 0.01 & no \\
18 & 2.61 & 0.034 & 0.01 & 0.07 & 1.00 & $1 / 30$ & 0.0075 & no \\
19 & 2.61 & 0.101 & 0.04 & 0.12 & 1.00 & $1 / 30$ & 0.0025 & no \\
20 & 2.61 & 0.252 & 0.10 & 0.19 & 1.00 & $1 / 30$ & 0.001 & no \\
21 & 0.26 & 0.025 & 0.10 & 0.61 & 0.01 & $1 / 3$ & 0.01 & no \\
22 & 0.26 & 0.034 & 0.13 & 0.70 & 0.01 & $1 / 3$ & 0.0075 & no \\
23 & 0.26 & 0.046 & 0.18 & 0.82 & 0.01 & $1 / 3$ & 0.0055 & no \\
24 & 0.26 & 0.101 & 0.39 & 1.22 & 0.01 & $1 / 3$ & 0.0025 & yes \\
25 & 0.26 & 0.252 & 0.97 & 1.92 & 0.01 & $1 / 3$ & 0.001 & yes \\
26 & 0.52 & 0.101 & 0.19 & 0.61 & 0.01 & $1 / 6$ & 0.0025 & yes \\
27 & 0.52 & 0.084 & 0.16 & 0.56 & 0.01 & $1 / 6$ & 0.0027 & no \\
28 & 0.52 & 0.093 & 0.18 & 0.59 & 0.01 & $1 / 6$ & 0.0030 & no \\
29 & 2.61 & 0.025 & 0.01 & 0.06 & 0.01 & $1 / 30$ & 0.01 & no \\
30 & 2.61 & 0.034 & 0.01 & 0.07 & 0.01 & $1 / 30$ & 0.0075 & no \\
31 & 2.61 & 0.050 & 0.02 & 0.09 & 0.01 & $1 / 30$ & 0.005 & no \\
32 & 2.61 & 0.101 & 0.04 & 0.12 & 0.01 & $1 / 30$ & 0.0025 & no \\
33 & 2.61 & 0.252 & 0.10 & 0.19 & 0.01 & $1 / 30$ & 0.001 & no \\
34 & 2.61 & 0.505 & 0.19 & 0.27 & 0.01 & $1 / 30$ & 0.0005 & no \\
35 & 2.61 & 0.561 & 0.21 & 0.29 & 0.01 & $1 / 30$ & 0.00045 & no \\
36 & 2.61 & 0.631 & 0.24 & 0.29 & 0.01 & $1 / 30$ & 0.0004 & yes \\
\hline & & & & & & & &
\end{tabular}




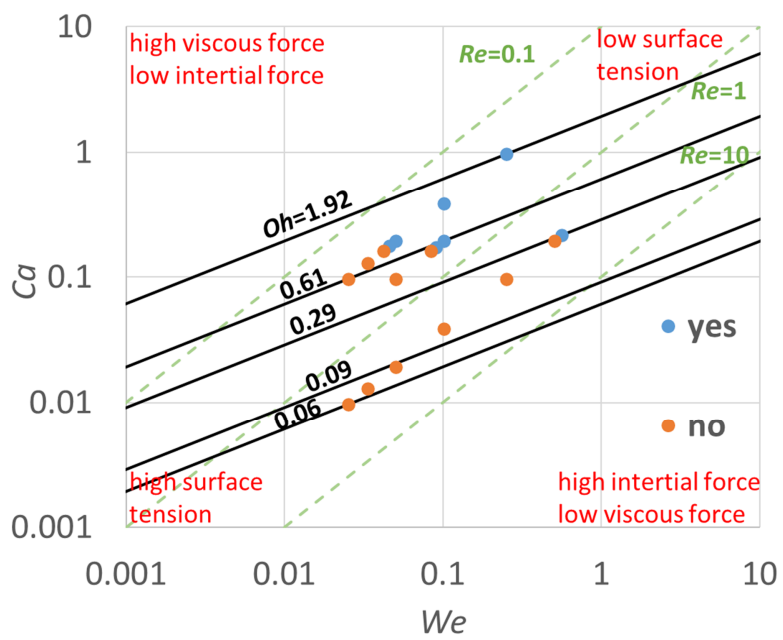

(a)

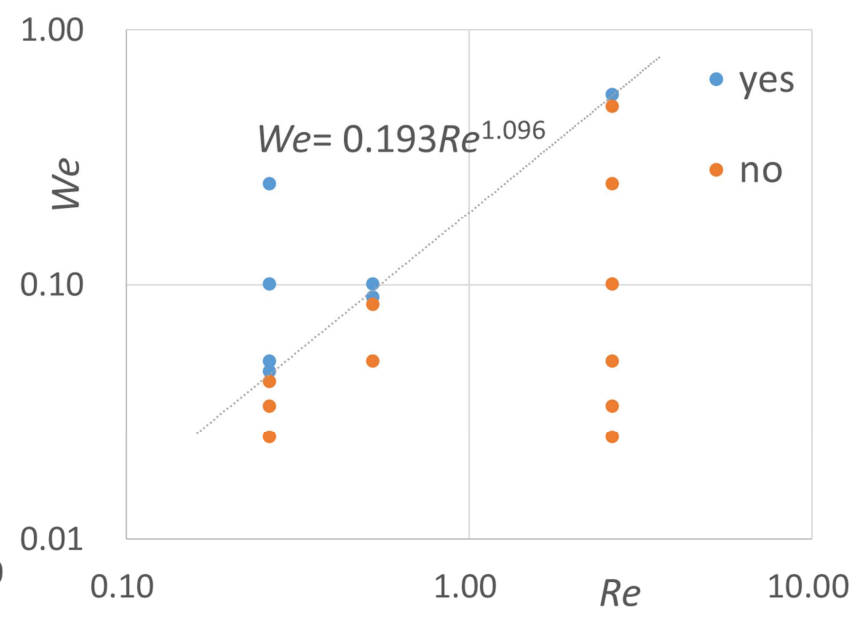

(b)

Fig. 16 (a) Phase chart at a viscosity ratio of 1.00 (b) The drop breakup conditions at a viscosity ratio of 1.00. 'Yes' indicates drop breakup. 'No' means drop did not break

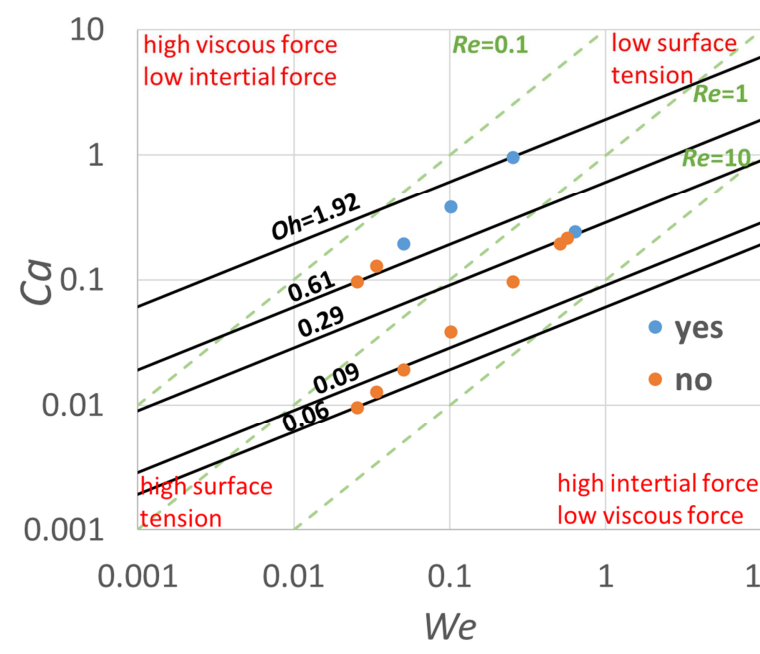

(a)

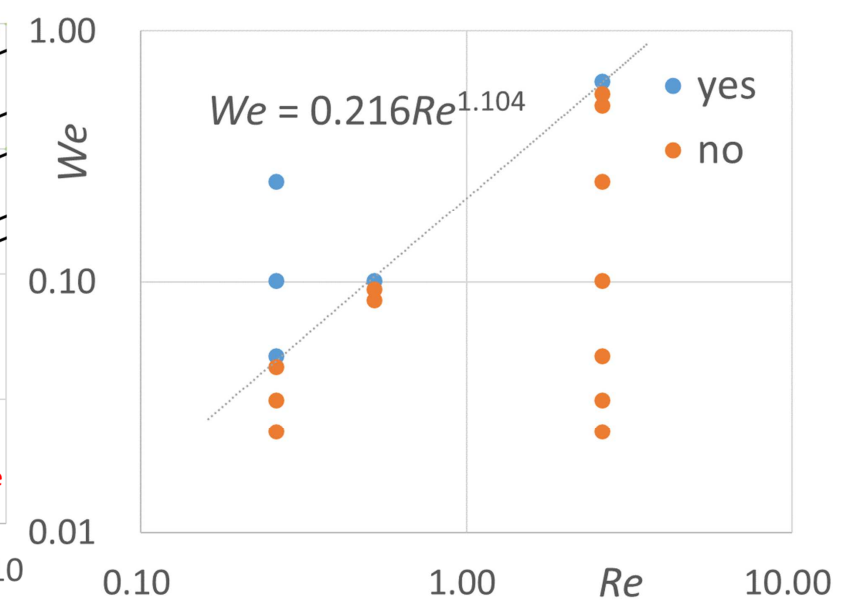

(b)

Fig. 17 (a) Phase chart at a viscosity ratio of 0.01 (b) The drop breakup conditions at a viscosity ratio of 0.01 . 'Yes' indicates drop breakup. 'No' means drop did not break 


\section{Conclusions}

In this paper, numerical simulations of the motion of Newtonian liquid drops flowing through a single pore in a microchannel as a result of the continuous flow of another immiscible liquid have been presented. We used the conservative phase-field lattice Boltzmann method to perform transient three-dimensional simulations.

The numerical code is verified and validated by a series of benchmark cases. The recovery of Laplace pressure is tested, and the deviation between numerical and theoretical predictions is within 3\%. Four cases of the layered flow of two immiscible liquids are performed to confirm our method can simulate the flow with viscosity ratio up to 1000 . Cases of recovery of the contact angle at the curved surface are conducted and obtained consistent results. The effect of the mesh resolution is investigated using the gravity-driven motion of a drop in ambient liquid in a channel, and it indicates that under the present circumstances 32 lu is enough for the drop diameters.

Numerical simulation of a drop falling through a pore due to gravity in an ambient continuous liquid phase is conducted and the results are in qualitatively good agreement compared to the experimental data of Ansari et al. (2018, 2019). In addition, quantitative verifications confirmed that the pressure distribution inside the deforming drop is consistent with the drop shape as it passes through the pore.

The influence of the surface tension, the average velocity of the continuous flow, and the viscosity ratio on the drop breakup were discussed in this paper. Smaller surface tension (high Weber number) promotes drop breakup when it passes through the pore throat. Lower Reynold number increases the probability of drop breakup. Drops of higher viscosity than the continuous phase break easier because of lower relative velocity in conjunction with interaction with the solid pore walls. Also, the coalescence of satellite drops is observed during this process. Finally, we show a $\mathrm{Ca}$ versus $\mathrm{We}$ phase chart to discuss the relative importance of the forces in this system leading to drop deformation and possibly breakup. We find that the lower surface tensions and larger viscous forces that dominate inertial forces are the key factors for the breakup. At 
the same time, a clear virtual dividing line is found in the We versus $R e$ map that distinguishes breakup from non-breakup. At constant $R e$, the critical We is smaller for the more viscous drop to break.

In the future work, we will focus on the drop breakup and coalescence in realistic porous media. We will also conduct experiments in porous media to visualize the multiphase flow to validate simulations quantitatively.

\section{Acknowledgements}

The authors appreciatively acknowledge the financial support from the National Key Research and Development Program of China (No.2016YFB0302801) and the China Scholarship Council. This research has been enabled by the use of computing resources provided by Compute Canada.

\section{Appendix A}

The discrete velocity set for $\mathrm{D} 3 \mathrm{Q} 27$ used for $f$ population is defined as follows:

$$
\left(\begin{array}{l}
e_{x} \\
e_{y} \\
e_{z}
\end{array}\right)=\left[\begin{array}{ccccccccccccccccccccccccccc}
0 & 1 & -1 & 0 & 0 & 0 & 0 & 1 & -1 & 1 & -1 & 1 & -1 & 1 & -1 & 1 & -1 & 1 & -1 & 1 & -1 & 1 & -1 & 0 & 0 & 0 & 0 \\
0 & 0 & 0 & 1 & -1 & 0 & 0 & 1 & 1 & -1 & -1 & 1 & 1 & -1 & -1 & 1 & 1 & -1 & -1 & 0 & 0 & 0 & 0 & 1 & -1 & 1 & -1 \\
0 & 0 & 0 & 0 & 0 & 1 & -1 & 1 & 1 & 1 & 1 & -1 & -1 & -1 & -1 & 0 & 0 & 0 & 0 & 1 & 1 & -1 & -1 & 1 & 1 & -1 & -1
\end{array}\right]
$$

And the corresponding weights are:

$$
\omega_{\alpha}=\frac{1}{216} \begin{cases}64, & \alpha=0 \\ 16, & \alpha=1-6 \\ 1, & \alpha=7-14 \\ 4, & \alpha=15-26\end{cases}
$$

The discrete velocity set for D3Q15 used for $g$ population is defined as follows:

$$
\left(\begin{array}{l}
e_{x} \\
e_{y} \\
e_{z}
\end{array}\right)=\left[\begin{array}{ccccccccccccccc}
0 & 1 & -1 & 0 & 0 & 0 & 0 & 1 & -1 & 1 & -1 & 1 & -1 & 1 & -1 \\
0 & 0 & 0 & 1 & -1 & 0 & 0 & 1 & 1 & -1 & -1 & 1 & 1 & -1 & -1 \\
0 & 0 & 0 & 0 & 0 & 1 & -1 & 1 & 1 & 1 & 1 & -1 & -1 & -1 & -1
\end{array}\right]
$$

And the corresponding weights are:

$$
\omega_{\alpha}=\frac{1}{72} \begin{cases}16, & \alpha=0 \\ 8, & \alpha=1-6 \\ 1, & \alpha=7-14\end{cases}
$$




\section{Appendix B}

The WMRT transformation matrix for D3Q27 is given as:

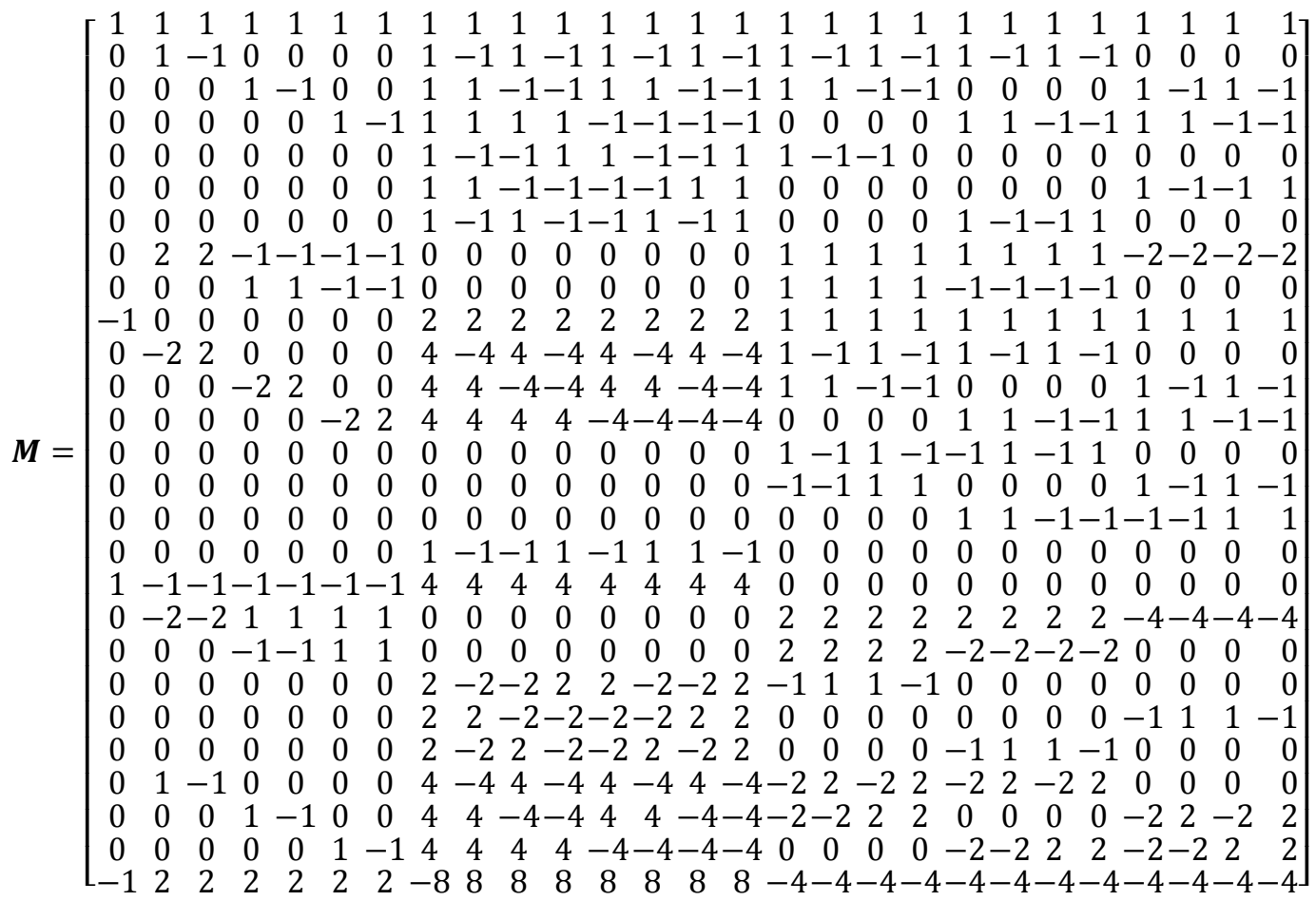

\section{Appendix C}

The L 2 convergence analysis of the layered flow of two immiscible liquids at $\eta=100$ is shown in Fig. 18. For the four cases, the channel heights were equal to 20, 30, 40 and 80 lu, respectively. The L2 error as a function of the mesh size is shown at a log-log plot in Fig.18. Second-order convergence is observed

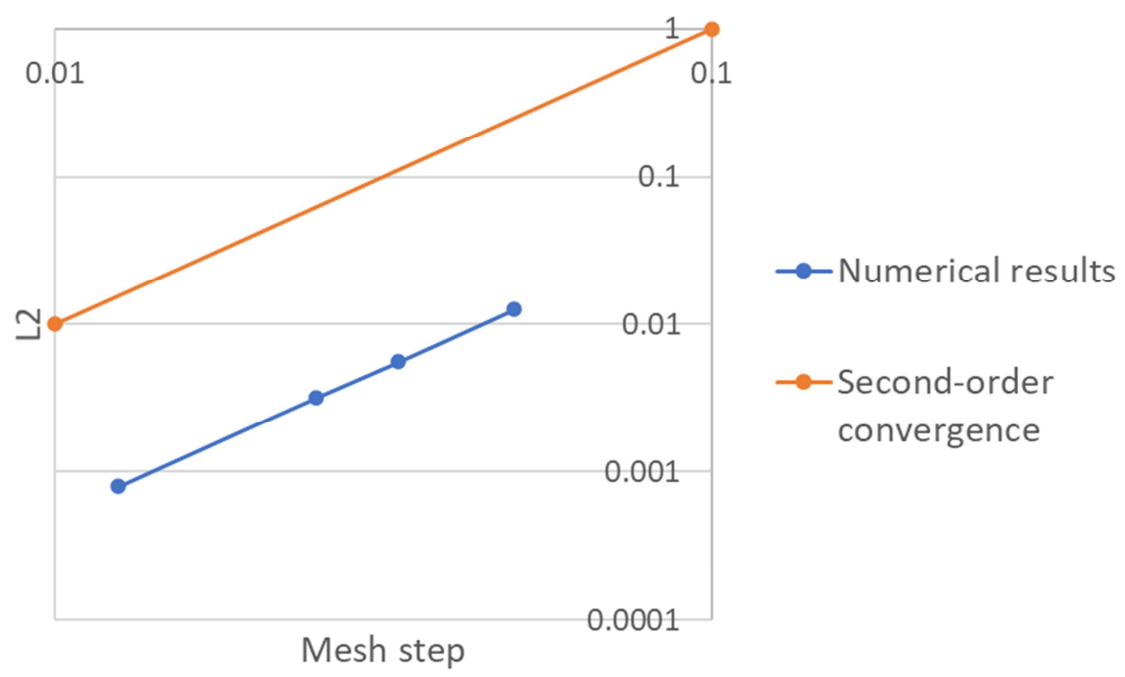

Fig.18 L2 Error vs Mesh size at $\eta=100$ 


\section{Appendix D}

Mesh sensitivity analysis concerning flow resolution was performed for Case 1 . The velocity profiles at $\bar{t}=9.33$ along the line $y=Y / 2$ in the $x=X / 2$ plane obtained using different mesh are shown in Fig. 19. The gravitational acceleration is set to $-3.38 \times 10^{-5},-1.0 \times 10^{-5}$, and $-2.96 \times 10^{-6}$ lu to achieve the same Reynold number for droplet diameters $\mathrm{D}=21,32$, and $48 \mathrm{lu}$, respectively (the dimensions of the domain are $27 \times 81 \times 180,40 \times 120 \times 270$ and $60 \times 180 \times 405$ lu). Velocity is non-dimensionalized as $\bar{u}=u / \sqrt{g D}$. The mesh with $\mathrm{D}=32$ lu has reached mesh independence because the results have good agreement with $\mathrm{D}=48 \mathrm{lu}$.

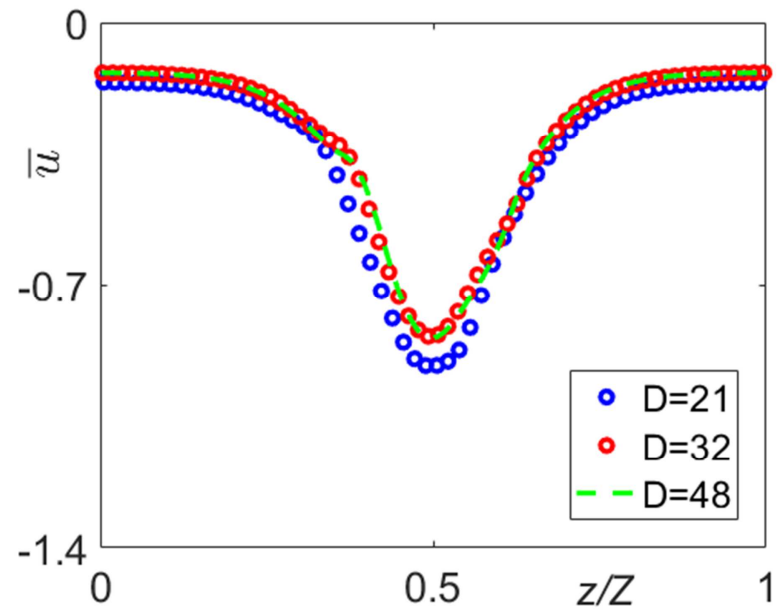

Fig. 19 The velocity profiles along the line $y=Y / 2$ in the $x=X / 2$ plane obtained on different mesh

\section{References}

Adler, P.M., Brenner, H., 1988. Multiphase Flow in Porous Media. Ann. Rev. Fluid Mech. 20, 35-59.

Ansari, S., Yusuf, Y., Sabbagh, R., Soltani, H., Kinsale, L., Nobes, D.S., 2018. An imaging derivation of the pressure field of a multi-phase flow in a porous media using $\mu$-SPIV. 19th Int. Symp. Appl. Laser Imaging Tech. to Fluid Mech.

Ansari, S., Yusuf, Y., Sabbagh, R., Nobes, D.S., 2019. Determining the pressure distribution of a multi-phase flow through a pore space using velocity measurement and shape analysis. Meas. Sci. Technol. 30, 054004.

Bhatnagar, P.L., Gross, E.P., Krook, M., 1954. A model for collision processes in gases. I. Small amplitude processes in charged and neutral one-component systems. Phys. Rev. 94, 511-525.

Cerdeira, A.T.S., Campos, J.B.L.M., Miranda, J.M., Araújo, J.D.P., 2020. Review on microbubbles and microdroplets flowing through microfluidic geometrical elements. Micromachines 11, 201.

Chai, L., Wang, L., Zhou, M., Xia, G., 2015. Two-phase flow pattern and pressure drop in silicon multi-microchannel with expansion-constriction cross-section. Exp. Therm. Fluid Sci. 60, 241-251.

Chio, H., Jensen, M.J., Wang, X., Bruus, H., Attinger, D., 2006. Transient pressure drops of gas bubbles passing through liquid-filled microchannel contractions: an experimental study. J. Micromech. Microeng. 16, 143-149.

Chiu, P.H., Lin, Y.T., 2011. A conservative phase field method for solving incompressible two-phase flows. J. Comput. Phys. 230, 185-204.

Chung, C., Hulsen, M.A., Kim, J.M., Ahn, K.H., Lee, S.J., 2008. Numerical study on the effect of viscoelasticity on drop deformation in simple shear and 5:1:5 planar contraction/expansion microchannel. J. Nonnewton. Fluid Mech. 155, $80-93$. 
Fakhari, A., Bolster, D., 2017. Diffuse interface modeling of three-phase contact line dynamics on curved boundaries: A lattice Boltzmann model for large density and viscosity ratios. J. Comput. Phys. 334, 620-638.

Fakhari, A., Bolster, D., Luo, L.S., 2017a. A weighted multiple-relaxation-time lattice Boltzmann method for multiphase flows and its application to partial coalescence cascades. J. Comput. Phys. 341, 22-43.

Fakhari, A., Mitchell, T., Leonardi, C., Bolster, D., 2017b. Improved locality of the phase-field lattice-Boltzmann model for immiscible fluids at high density ratios. Phys. Rev. E 96, 053301.

Harvie, D.J.E., Davidson, M.R., Cooper-White, J.J., Rudman, M., 2006. A parametric study of droplet deformation through a microfluidic contraction: Low viscosity Newtonian droplets. Chem. Eng. Sci. 61, 5149-5158.

Harvie, D.J.E., Davidson, M.R., Cooper-White, J.J., Rudman, M., 2005. A parametric study of droplet deformation through a microfluidic contraction. ANZIAM J. 46, 150-166.

Hellou, M., Vo, T.T.G., 2015. Motion of non-wetting drop in constricted geometry. Mech. Res. Commun. 70, 85-93.

Hemmat, M., Borhan, A., 1996. Buoyancy-driven motion of drops and bubbles in a periodically constricted capillary. Chem. Eng. Commun. 148-150, 363-384.

Izbassarov, D., Muradoglu, M., 2016. A computational study of two-phase viscoelastic systems in a capillary tube with a sudden contraction/expansion. Phys. Fluids 28, 012110.

Jacob, B., 2018. Theory and Applications of Transport in Porous Media Modeling Phenomena of Flow and Transport in Porous Media. Springer, Switzerland.

Jacqmin, D., 2000. Contact-line dynamics of a diffuse fluid interface. J. Fluid Mech. 402, 57-88.

Jensen, M.J., Goranović, G., Bruus, H., 2004. The clogging pressure of bubbles in hydrophilic microchannel contractions. J. Micromech. Microeng. 14, 876-883.

Kim, S., Wang, H., Yan, L., Zhang, X., Cheng, Y., 2020. Continuous preparation of itraconazole nanoparticles using droplet-based microreactor. Chem. Eng. J. 393, 124721.

Komrakova, A.E., Shardt, O., Eskin, D., Derksen, J.J., 2015. Effects of dispersed phase viscosity on drop deformation and breakup in inertial shear flow. Chem. Eng. Sci. 126, 150-159.

Komrakova, A.E., Shardt, O., Eskin, D., Derksen, J.J., 2014. Lattice Boltzmann simulations of drop deformation and breakup in shear flow. Int. J. Multiph. Flow 59, 24-43.

Lee, G.W., Shim, G.H., Kim, J.M., Seol, C., Kim, J.H., Kim, S.M., Ahn, H.S., 2020. Two/three-dimensional reduced graphene oxide coating for porous flow distributor in polymer electrolyte membrane fuel cell. Int. J. Hydrogen Energy 45, 12972-12981.

Li, D., Mao, D., Li, Q., Chen, Y.S., Chen, X., Xu, X., 2018. A versatile porous 3D polyurethane/polyacrylic acid (PU-PAA) membrane for one-step multiple contaminants water purification. J. Memb. Sci. 563, 191-198.

Mitchell, T., Leonardi, C., Fakhari, A., 2018. Development of a three-dimensional phase-field lattice Boltzmann method for the study of immiscible fluids at high density ratios. Int. J. Multiph. Flow 107, 1-15.

Mulligan, M.K., Rothstein, J.P., 2011. The effect of confinement-induced shear on drop deformation and breakup in microfluidic extensional flows. Phys. Fluids 23, 022004.

Olbricht, W.L., Leal, L.G., 1983. The creeping motion of immiscible drops through a converging/diverging tube. J. Fluid Mech. 134, 329-355.

Olgac, U., Kayaalp, A.D., Muradoglu, M., 2006. Buoyancy-driven motion and breakup of viscous drops in constricted capillaries. Int. J. Multiph. Flow 32, 1055-1071.

Patel, T., Patel, D., Thakkar, N., Lakdawala, A., 2019. A numerical study on bubble dynamics in sinusoidal channels. Phys. Fluids 31, 052103.

Perazzo, A., Tomaiuolo, G., Preziosi, V., Guido, S., 2018. Emulsions in porous media: From single droplet behavior to applications for oil recovery. Adv. Colloid Interface Sci. 256, 305-325. 
Schulz, V.P., Abbaspour, N., Baumeister, T., Röder, T., 2019. Lattice-Boltzmann Simulation and Experimental Validation of a Microfluidic T-Junction for Slug Flow Generation. ChemEngineering 3, 48.

Sun, Y., Beckermann, C., 2007. Sharp interface tracking using the phase-field equation. J. Comput. Phys. 220, 626-653.

Telmadarreie, A., Doda, A., Trivedi, J.J., Kuru, E., Choi, P., 2016. $\mathrm{CO}_{2}$ microbubbles - A potential fluid for enhanced oil recovery: Bulk and porous media studies. J. Pet. Sci. Eng. 138, 160-173.

Zhao, X., 2007. Drop breakup in dilute Newtonian emulsions in simple shear flow: New drop breakup mechanisms. J. Rheol. 51, 367-392.

Zheng, X., Lu, J., Bai, B., Tryggvason, G., 2020. The passage of a bubble or a drop past an obstruction in a channel. Phys. Fluids 32, 023303.

Zinchenko, A.Z., Davis, R.H., 2017. Motion of Deformable Drops Through Porous Media. Annu. Rev. Fluid Mech. 49, 71-90.

Zu, Y.Q., He, S., 2013. Phase-field-based lattice Boltzmann model for incompressible binary fluid systems with density and viscosity contrasts. Phys. Rev. E 87, 043301. 